\title{
Invasion fitness, inclusive fitness, and reproductive numbers in heterogeneous populations
}

\author{
Laurent Lehmann ${ }^{\star} \quad$ Charles Mullon ${ }^{\dagger} \quad$ Erol Akçay $^{\ddagger} \quad$ Jeremy Van Cleve
}

\footnotetext{
${ }^{*}$ Department of Ecology and Evolution, University of Lausanne, Switzerland. (Laurent.lehmann@unil.ch)

${ }^{\dagger}$ Department of Ecology and Evolution, University of Lausanne, Switzerland. (Charles.Mullon@unil.ch)

${ }^{\ddagger}$ Department of Biology, University of Pennsylvania, USA. (eakcay@sas.upenn.edu)

${ }^{\S}$ Department of Biology, University of Kentucky, USA. (jvancleve@uky. edu)
} 


\begin{abstract}
How should fitness be measured to determine which phenotype or "strategy" is uninvadable when evolution occurs in subdivided populations subject to local demographic and environmental heterogeneity? Several invasion fitness measures, such as basic reproductive number, lifetime dispersal success of a local lineage, or inclusive fitness have been proposed to address this question, but the relationships between them and their generality remains unclear. Here, we ascertain uninvadability (all mutant strategies always go extinct) in terms of the growth rate of a mutant allele arising as a single copy in a population. We show from this growth rate that uninvadability is equivalently characterized by at least three conceptually distinct invasion fitness measures: (i) lineage fitness, giving the average personal fitness of a randomly sampled mutant lineage member; (ii) inclusive fitness, giving a reproductive value weighted average of the direct fitness cost and relatedness weighted indirect fitness benefits accruing to a randomly sampled mutant lineage member; and (iii) three types of reproductive numbers, giving lifetime success of a local lineage. Our analysis connects approaches that have been deemed different, generalizes the exact version of inclusive fitness to class-structured populations, and provides a biological interpretation of selection on a mutant allele under arbitrary strength of selection.
\end{abstract}

Keywords: growth rate, invasion fitness, inclusive fitness, reproductive number, invadability 


\section{Introduction}

It is well established (if perhaps unwelcome) that in general adaptiveness is not increased by short-term evolution (Moran, 1964; Eshel, 1991; Ewens, 2004). In contrast, when long-term evolution can be described by a substitution process where a population transitions from one fixed allele to another through the recurrent invasion of mutant alleles, the population may eventually evolve to an uninvadable state (i.e., a state that is resistant to invasion by any alternative strategy, Eshel, 1991, 1996; Hammerstein, 1996; Weissing, 1996; Van Cleve, 2015). An uninvadable strategy is "optimal" among a specified set of alternatives because it maximizes the growth rate of the underlying coding gene when the gene is rare (Eshel, 1991, 1996; Hammerstein, 1996; Weissing, 1996). Uninvadable strategies are thus adaptations (sensu Reeve and Sherman, 1993) and evolutionary invasion analysis has become a very successful approach to understand theoretically long term phenotypic evolution (e.g., Maynard Smith, 1982; Eshel and Feldman, 1984; Parker and Maynard Smith, 1990; Charlesworth, 1994; Metz et al., 1996; Ferrière and Gatto, 1995; McNamara et al., 2001; Lion and van Baalen, 2007; Metz, 2011; van Baalen, 2013).

When a mutant allele arises as a single copy in a population, its growth rate, $\rho$, determines in general whether the mutant allele will eventually go extinct or survive (Tuljapurkar, 1989; Metz et al., 1992; Rand et al., 1994; Charlesworth, 1994; Ferrière and Gatto, 1995; Caswell, 2000). Intuitively, the growth rate is a gene-centered measure of evolutionary success (sensu Dawkins, 1978). Technically, the growth rate is the dominant eigenvalue of a matrix determining the transitions between the different states in which the mutant allele can reside and describes the growth of a typical trajectory of the mutant lineage since its appearance as a single copy(Tuljapurkar, 1989; Tuljapurkar et al., 2003; Caswell, 2000; Ferrière and Gatto, 1995). Since evolutionary biologists often try to understand adaptations in terms of the fitness properties exhibited by individuals, such as survival and fecundity, it is important to understand the exact interpretation of the growth rate in terms of individual-centered fitness components. Interpreting the growth rate this way seems clear in panmictic populations. In the absence of genetic conflict within individuals, maximizing the growth rate amounts to maximizing the personal (lifetime) fitness of an individual, which is determined by its survival and fecundity schedules in stage-structured populations (Eshel and Feldman, 1984; Hammerstein, 1996; Weissing, 1996; Charlesworth, 1994; Caswell, 2000). This result relies on the assumption that mutants are rare, which allows one to neglect the interactions between individuals carrying the mutant allele in the invasion analysis. 
When dispersal is limited due to family or spatial population structure, interactions between mutants can no longer be neglected when evaluating the growth rate; mutant-mutant interactions will occur locally at the level of the interaction group even if the mutant is globally rare. Since the mutant is no longer necessarily locally rare, one needs to track groups with different numbers of mutant alleles (i.e., the local distribution of mutants). In this case, the growth rate $\rho$ becomes the eigenvalue of a matrix describing the transitions between different group states (Motro, 1982; Bulmer, 1986; van Baalen and Rand, 1998; Wild, 2011). In this case, the interpretation of the growth rate in terms of individual-centered fitness components is no longer straightforward. In order to understand exactly what the growth rate represents biologically, it needs to be unpacked and expressed in terms of individual-centered properties. Until now, no general interpretation of the mutant growth rate has been provided for group structured populations subject to local heterogeneities, such as demographic or environmental fluctuations.

In the absence of a general and clear interpretation of the growht rate of a mutant allele, several different measures of invasion fitness, defined as any quantity allowing to determine the fate of a mutant, have been proposed. One approach computes invasion fitness as the basic reproductive number, $R_{0}$, of a mutant lineage (Massol et al., 2009). This gives the total number of successful emigrants produced by a mutant lineage over its lifetime when the lineage was started in a single group by some distribution of emigrants. It is well established in mathematical biology that maximizing the basic reproductive number $R_{0}$ (the eigenvalue of the next generation matrix associated with the process) is equivalent to maximizing its growth rate (holding the resident population constant), and thus predicts the direction of selection in the same way (Caswell, 2000; Ellner and Rees, 2006).

A closely related approach puts forward the total number of successful emigrants produced by a mutant lineage over its lifetime in a single group that was founded by a single emigrant, called $R_{\mathrm{m}}$, as the appropriate measure of invasion fitness (Metz and Gyllenberg, 2001; Cadet et al., 2003). By assumption, this requires that individuals disperse independently and not in clusters, which excludes propagule dispersal. However, a fitness measure should in general be able to account for propagule dispersal, which is important for understanding the life cycle of many species. This raises the question of the general connection between $R_{0}$ and $R_{\mathrm{m}}$ and their interpretation in terms of individual-centered fitness components.

Further, invasion fitness can also be computed as the personal fitness of a randomly sampled carrier of the mutant allele from the founding lineage (Day, 2001; Lehmann et al., 2015; Mullon et al., 2016), which we refer as lineage fitness. In contrast to $R_{0}$ and $R_{\mathrm{m}}$, lineage fitness is expressed in terms of individual- 
centered fitness components, but it has not yet been generalized to subdivided populations with local heterogeneities.

Among all alternative methods for studying evolution in structured populations, the most popular one, however, has perhaps been the direct fitness method of social evolution theory (e.g., Taylor and Frank, 1996; Frank, 1998; Rousset, 2004; Wenseleers et al., 2010). This approach quantifies the effect on selection of local interactions between individuals carrying a mutant allele by using relatedness coefficients and ascertains the direction of selection on a mutant lineage by way of the inclusive fitness effect. The inclusive fitness effect is a weak selection decomposition of the change in the personal fitness of a randomly sampled carrier of the mutant allele into direct effects, resulting from an individual expressing the mutant instead of the resident allele, and indirect effects weighted by relatedness among group members, resulting from group neighbours expressing the mutant. The inclusive fitness effect has helped understand the selection pressure on very diverse phenotypes including the sex-ratio, reproductive effort, genomic imprinting, dispersal, menopause, parasite virulence, interactive behavior, senescence, and niche construction in groups structured populations (e.g., Taylor, 1988; Haig, 1997; Frank, 1998; Gandon, 1999; Taylor and Irwin, 2000; Pen, 2000; Lehmann, 2008; Wild et al., 2009; Lion and Gandon, 2009; Johnstone and Cant, 2010; Ronce and Promislow, 2010; Akçay and Van Cleve, 2012; Lion, 2013).

Despite their apparent differences, inclusive fitness, lineage fitness, or, more generally invasion fitness measures, are in fact tightly connected (Akçay and Van Cleve, 2016). For example, under constant demography, the inclusive fitness effect amounts to evaluating the sensitivity of the number of emigrants $R_{\mathrm{m}}$ or the growth rate $\rho$ with respect to variation in continuous trait values and lineage fitness is equal to $\rho$ (Ajar, 2003; Lehmann et al., 2015; Mullon et al., 2016), but the general connection between mutant growth rates, inclusive fitness, lineage fitness, and the reproductive numbers, has not been worked out under arbitrary mutant trait types and selection strength with local demographic and/or environmental heterogeneities.

The aim of this paper is to fill these gaps by providing a general interpretation of the mutant growth rate in terms of individual-centered fitness components and connecting formally to each other the different invasion fitness measures. Our results highlight the conceptual unity underlying invasion fitness and resolve some long standing about how inclusive fitness fits in under arbitrary mutant type and strength of selection. 


\section{Model}

\section{Life-cycle}

We consider a population of haploid individuals divided into an infinite number of groups. The population is censused at discrete time demographic periods. In each period, each group, independently from each other, can be in one of a countable number of demographic-environmental states. A state can determine the number of individuals in a group ("demographic" state) and/or any environmental factor affecting all individuals within a group ("environmental" state). Local state fluctuations in the population due to demographic or environmental processes can result in population level patterns of temporal and spatial heterogeneity.

Dispersal may occur between groups by individuals alone or by groups of individuals (i.e., propagule dispersal), but dispersal is always assumed to be uniform between groups in the population; in other words, we consider an island model of dispersal (Wright, 1931). The model allows us to represent classical metapopulation processes with variable local group sizes (Chesson, 1981; Rousset and Ronce, 2004), insect colony dynamics with endogenous growth (Avila and Fromhage, 2015), as well as compartmentalized replication as occurs in the stochastic corrector model for prebiotic evolution (Szathmary and Demeter, 1987; Grey et al., 1995).

We assume that only two alleles can segregate in the population, a mutant allele with type $\tau$ and a resident allele of type $\theta$ where the set of all possible types is called $\Theta$. Suppose that initially the population is monomorphic or fixed for the resident type $\theta$ and that a single individual mutates to type $\tau$. Will the mutant "invade" the population and increase in frequency? If the resident type $\theta$ is such that any mutant type $\tau \in \Theta$ goes extinct with probability one, we will say that $\theta$ is uninvadable. A state that is uninvadable is an evolutionarily stable state. Our aim is to characterize uninvadability mathematically and biologically.

\section{The resident demographic equilibrium}

Following standard assumptions for the dynamics of mutant-resident substitutions (Eshel and Feldman, 1984; Eshel, 1996; Hammerstein, 1996; Weissing, 1996; Metz et al., 1996), we assume that a mutant can only arise in a resident population that is at its demographic equilibrium, and we start by characterizing this equilibrium. Our main assumption is that the stochastic process describing the state dynamics of a 
focal group in the resident population is given by a discrete time Markov chain on a countable state space (Karlin and Taylor, 1975; [osifescu, 2007), where the time scale is that of a demographic period (i.e., the scale at which births, deaths, dispersal, and other events occur).

Because groups may affect each other demographically through dispersal, the transition probabilities for this Markov chain may depend endogenously on the resident population dynamics. But since there is an infinite number of groups, the set of infinite interacting Markov chains (one for each group) can be described as a single (inhomogeneous) Markov chain, whose transition probabilities are functions of the expected value of the process (Chesson, 1981, 1984). We assume that this Markov chain is regular, irreducible and aperiodic (Karlin and Taylor, 1975; [osifescu, 2007), and thus has a stationary distribution (see Appendix A).

\section{The mutant multitype branching process}

We now introduce a mutant into the backdrop of the resident population in its stationary demographic regime. Denote by $M_{t}(s, i)$ the random number of groups in the population that are in state $s \in S$ and have exactly $i \in I(s)=\{1,2, \ldots, n(s)\}$ mutant individuals at time $t=0,1,2, \ldots$ where $n(s)$ is the number of individuals in a group in state $s$ and $t=0$ is the time of appearance of the mutant. Denote by $\mathbf{M}_{t}=$ $\left(M_{t}(s, i)\right)_{s \in S, i \in I(s)}$ the vector collecting the $M_{t}(s, i)$ random variables and $\mathbf{e}_{s}$ a vector of the same dimension but whose $(s, 1)$-th component is equal to one, otherwise zero. Starting with a single initial mutant of type $\tau$ in a focal group in state $s$ at time $t=0$, namely $\mathrm{M}_{0}=\mathrm{e}(s)$, we are interested in finding a necessary and sufficient condition for the mutant type $\tau$ to go extinct in finite time with probability one for any state $s \in \mathcal{S}$ (formally, a condition for $\operatorname{Pr}\left[\mathbf{M}_{t}=\mathbf{0}\right.$ for some $\left.t \in \mathbb{N} \mid \mathbf{M}_{0}=\mathbf{e}(s)\right]=1$ for all $s \in \mathcal{S}$ ).

Since we are interested only in characterizing extinction of the mutant, we assume that it will always remain rare in the total population and approximate the mutant stochastic process as a multitype branching process (Harris, 1963; Karlin and Taylor, 1975; Wild, 2011). It is then sufficient to focus on the (regular) matrix A whose $\left(s^{\prime}, i^{\prime} ; s, i\right)$ element, denoted $a\left(s^{\prime}, i^{\prime} \mid s, i\right)$, is the expected number of groups in state $\left(s^{\prime}, i^{\prime}\right)$ that are "produced" over one demographic time period by a focal group in state $(s, i)$ when the population is otherwise monomorphic for $\tau$. It is useful to decompose this as

$$
a\left(s^{\prime}, i^{\prime} \mid s, i\right)=p\left(s^{\prime}, i^{\prime} \mid s, i\right)+d\left(s^{\prime}, i^{\prime} \mid s, i\right),
$$


which consists of two terms representing two distinct biological processes. The first is the intra-group (or intra-compartmental) change described by the transition probability $p\left(s^{\prime}, i^{\prime} \mid s, i\right)$ that a focal group in state $(s, i)$ turns into a group in state $\left(s^{\prime}, i^{\prime}\right)$ after one demographic time period. The second process is the success of a group in replacing other groups by reproduction or fission, which is represented by $d\left(s^{\prime}, i^{\prime} \mid s, i\right)$ that measures the expected number of groups in state $\left(s^{\prime}, i^{\prime}\right)$ produced by emigration from, or fission, of a focal group of state $(s, i)$. By "producing" a group of state $\left(s^{\prime}, i^{\prime}\right)$, we mean that for a metapopulation process a focal group in state $(s, i)$ in a parental generation leaves $i^{\prime} \in I\left(s^{\prime}\right)$ mutant offspring in a group that will be in state $s^{\prime}$ after one demographic time period. For compartmental replication processes (e.g., Grey et al., 1995) this means producing a group in state $\left(s^{\prime}, i^{\prime}\right)$.

\section{Invasion fitness}

It follows from standard results on multitype branching processes (Harris, 1963; Karlin and Taylor, 1975) that the lineage descending from a single mutant $\tau$ arising in any of the demographic state of the resident $\theta$ population, will go extinct with probability one if the leading eigenvalue $\rho(\tau, \theta)$ of $\mathbf{A}(\tau, \theta)$ is less than or equal to 1 . Namely, extinction with probability one occurs if and only if

$$
\rho(\tau, \theta) \leq 1,
$$

where $\rho$ satisfies

$$
\rho(\tau, \theta) \mathbf{u}(\tau, \theta)=\mathbf{A}(\tau, \theta) \mathbf{u}(\tau, \theta)
$$

and $\mathbf{u}(\tau, \theta)$ is the leading right eigenvector of $\mathbf{A}(\tau, \theta)$.

The interpretation of $\rho(\tau, \theta)$ is that it gives the asymptotic growth rate of an average trajectory of a mutant lineage; that is, the collection of individuals descending from an individual in which the mutation appeared (Cohen, 1979; Tuljapurkar et al., 2003). In the long-run, the average mutant lineage grows in the direction of $\mathbf{u}(\tau, \theta)$ so that this vector can be interpreted as a quasi-stationary distribution of group genetic-demographic-environmental states containing at least one individual belonging to the mutant lineage. Namely, element $(s, i)$ of $\mathbf{u}$, that is $u(s, i)$, is the asymptotic frequency of $s$-type groups with $i \geq 1$ mutants; this interpretation holds whether the mutant lineage goes extinct or invades the population (Har- 
ris, 1963).

It follows directly from the construction of the model that $\rho(\theta, \theta)=1$; namely, the growth of a resident lineage in a resident population is equal to one (see Appendix A for a proof). This implies that a resident type $\theta \in \Theta$ is uninvadable if, and only if,

$$
\rho(\tau, \theta) \leq 1 \quad \forall \tau \in \Theta
$$

Thus $\theta$ is uninvadable only if $\theta$ solves the maximization problem $\max _{\tau \in \Theta} \rho(\tau, \theta)$.

Now that we have a mathematical characterization of uninvadability in terms of the growth rate $\rho(\tau, \theta)$ of the mutant lineage, we present five different measures of invasion fitness that are all related to $\rho(\tau, \theta)$ and are all expressed in term of biological quantities that have appeared previously in the literature. All these quantities are derived in the Appendix from the elements $a\left(s^{\prime}, i^{\prime} \mid s, i\right), p\left(s^{\prime}, i^{\prime} \mid s, i\right)$, and/or $d\left(s^{\prime}, i^{\prime} \mid s, i\right)$ (eq. (11)), and the explicit mathematical expressions are given in Table 1 .

\section{An ecstasy in five fits: five invasion fitness measures}

\section{Lineage fitness}

First, we let the lineage fitness of a mutant type $\tau$ in a resident $\theta$ population be

$$
W(\tau, \theta)=\sum_{s^{\prime} \in S} \sum_{s \in S} \sum_{i \in I(s)} w\left(s^{\prime} \mid s, i\right) q(i \mid s) q(s),
$$

where $w\left(s^{\prime} \mid s, i\right)$ is the expected number of successful offspring, which settle in groups of type $s^{\prime}$, given that the parent is a mutant residing in a group in state $(s, i)$. Lineage fitness also depends on the probability $q(i \mid$ $s$ ) that, conditional on being sampled in a group in state $s$, a randomly sampled mutant individual from the mutant lineage has $i-1$ mutant neighbors. This can be thought as the conditional mutant experienced profile distribution in the stationary mutant distribution, and $q(s)$ is the probability that a randomly sampled individual from the mutant lineage finds itself in a group in state $s$. When there is only one demographic state, $W(\tau, \theta)$ reduces to eq. (A.1) of Day (2001) and eq. (A.7) of Mullon et al. (2016).

Lineage fitness $W(\tau, \theta)$ is the fitness of a randomly sampled carrier of the mutant allele from its lineage, where $w\left(s^{\prime} \mid s, i\right)$ is an individual-centered fitness component variously called "direct", "personal", or 
"individual" fitness in social evolutionary theory (e.g, Frank, 1998; Rousset, 2004), and will be here refered to it as personal fitness. It involves offspring reaching adulthood in the group of the parent and in other groups through dispersal, and can thus also be written as

$$
w\left(s^{\prime} \mid s, i\right)=w_{\mathrm{p}}\left(s^{\prime} \mid s, i\right)+w_{\mathrm{d}}\left(s^{\prime} \mid s, i\right) .
$$

Here, $w_{\mathrm{p}}\left(s^{\prime} \mid s, i\right)$ is the expected number of philopatric offspring, which settle in a group in state $s^{\prime}$, given that the parent is a mutant that reproduced in a group in state $(s, i)$, while $w_{\mathrm{d}}\left(s^{\prime} \mid s, i\right)$ is such offspring produced by dispersal, and thus reach adulthood in other groups in state $s^{\prime}$. This decomposition of personal fitness matches the decomposition of the element of the transition matrix of the mutant given in eq. (1D)

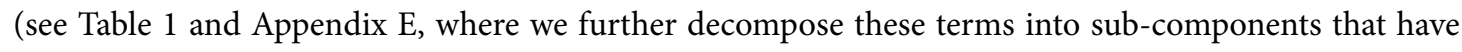
appeared previously in the literature).

In Appendix $\mathbb{B}$, we show that the growth rate of the mutant lineage is exactly equal to lineage fitness of the mutant; namely,

$$
W(\tau, \theta)=\rho(\tau, \theta) .
$$

This equation immediately implies that $\tau$ is uninvadable if it solves the optimization problem $\max _{\tau \in \Theta} W(\tau, \theta)$. In other words, the type is uninvadable if it "maximizes" lineage fitness. Since lineage fitness is the statistical average over all genetic demographic-environmental states of the personal fitness of the carrier of the mutant allele, it can be interpreted as a gene-centered measure of fitness $\$$, since it is the maximand of the number of mutant replica copies produced by a representative individual carrying the mutant allele. The condition for uninvadability (eq. 7) can also be interpreted as a version for class structured population of the seminal uninvadability condition obtained for multilocus systems in panmictic populations, where the statistical average is over multilocus genetic states (Eshel and Feldman, 1984, eq. 10, Eshel et al., 1998, eq. 7).

\footnotetext{
${ }^{1} \mathrm{EA}$ and JVC prefer the nomenclature "gene-lineage-centered" (Akçay and Van Cleve, 2016).
} 


\section{Inclusive fitness}

Let us now decompose personal fitness as

$$
w\left(s^{\prime} \mid s, i\right)=w^{\circ}\left(s^{\prime} \mid s\right)-\gamma\left(s^{\prime} \mid s\right)+\beta\left(s^{\prime} \mid s\right)\left(\frac{i-1}{n(s)-1}\right)+\epsilon_{i \prime}
$$

where $w^{\circ}\left(s^{\prime} \mid s\right)$ is the expected number of successful offspring, which settle in groups of type $s^{\prime}$, given that the parent is a resident reproducing in a group in state $s$ in a monomorphic resident population, and where the superscript $\circ$ will throughout denote a quantity that is evaluated in the absence of natural selection, i.e., neutral process determined by the monomorphic resident population. Personal fitness also depends on $\gamma\left(s^{\prime} \mid s\right)$, which is the additive effect on the personal fitness of an individual stemming from it switching to the expression of the mutant allele, $\beta\left(s^{\prime} \mid s\right)$, which is the additive effect on the personal fitness of a mutant stemming from a neighbor switching to the expression of the mutant, and $(i-1) /(n(s)-1)$, which is the frequency of mutants in a the neighborhood of a mutant individual in a group with $i$ mutants. The direct effect $\gamma\left(s^{\prime} \mid s\right)$ and the indirect effect $\beta\left(s^{\prime} \mid s\right)$ are obtained by minimizing the mean squared error $\epsilon_{i}$ in the linear prediction of personal fitness (see Box 1 for details).

We let the inclusive fitness of a mutant type $\tau$ in a population with residents of type $\theta$ be

$$
W_{\mathrm{IF}}(\tau, \theta)=1+\sum_{s^{\prime} \in S} \sum_{s \in \mathcal{S}} v^{\circ}\left(s^{\prime}\right)\left[-\gamma\left(s^{\prime} \mid s\right)+\beta\left(s^{\prime} \mid s\right) r(s)\right] q(s)
$$

where $v^{\circ}(s)$ is the neutral reproductive value of a single individual reproducing in a group in state $s$. This is the relative asymptotic contribution of an individual in state $s$ to the population (see Taylor, 1996 and Rousset, 2004 for an introduction to this concept). Inclusive fitness also depends on the probability $r(s)$ that, conditional on being sampled in a group in state $s$, an individual carrying the mutant experiences a randomly sampled neighbour that also carries the mutant allele. This is a measure of pairwise relatedness between two individuals in a group (see Table 1). In a monomorphic resident population, relatedness [then given by $r^{\circ}(s)$ ] reduces to the standard concept of probability of identity by descent between two randomly sampled group members (e.g., Frank, 1998; Rousset, 2004). In sum, the inclusive fitness $W_{\mathrm{IF}}(\tau, \theta)$ of a randomly sampled mutant from the lineage distribution $q(s)$ is the reproductive-value weighted average personal fitness cost $\gamma\left(s^{\prime} \mid s\right)$ of carrying the mutant allele and the relatedness weighted personal indirect fitness benefit $\beta\left(s^{\prime} \mid s\right)$ of carrying the mutant. 
We show in Appendix 9 that inclusive fitness $W_{\mathrm{IF}}(\tau, \theta)$ predicts whether or not the mutant invades in the same way as the growth rate $\rho(\tau, \theta)$; that is,

$$
W_{\mathrm{IF}}(\tau, \theta) \leq 1 \Longleftrightarrow \rho(\tau, \theta) \leq 1
$$

Hence, a strategy is uninvadable if and only if inclusive fitness is maximized, in the sense that $\tau$ solves the problem $\max _{\tau \in \Theta} W_{\mathrm{IF}}(\tau, \theta)$. This shows that, regardless of the force of selection, uninvadability can be expressed in terms of the three standard measures of "value" emphasized by social evolution theory (Frank, 1998): (i) the direct cost and indirect benefit within each class of an individual expressing the mutant, (ii) the pairwise relatedness between interacting individuals, and (iii) the neutral reproductive value of the descendants in each class. It is important to note that the inclusive fitness $W_{\mathrm{IF}}(\tau, \theta)$ is not equal to the growth rate $\rho(\tau, \theta)$, but is a linear function of it (see eq. (C.5) in Appendix Q).

\section{Reproductive numbers}

We let the basic reproductive number of a mutant type $\tau$ in a resident $\theta$ population be

$$
R_{0}(\tau, \theta)=\frac{R_{\mathrm{m}}(\tau, \theta)}{N_{\mathrm{F}}(\tau, \theta)}
$$

which depends on the expected number $N_{\mathrm{F}}(\tau, \theta)$ of mutant colonizing the same group and descending from the same natal group, and on the successful number of emigrants

$$
R_{\mathrm{m}}(\tau, \theta)=\sum_{s^{\prime} \in \mathcal{S}} \sum_{s \in S} \sum_{i \in I(s)} w_{\mathrm{d}}\left(s^{\prime} \mid s, i\right) i \bar{t}(s, i)
$$

produced by all individuals of the mutant lineage over its lifetime in a single group. This depends on the expected number $w_{\mathrm{d}}\left(s^{\prime} \mid s, i\right)$ of emigrant offspring that settle in groups of type $s^{\prime}$ (see eq. (6)) and on the total expected amount of time $\bar{t}(s, i)$ that a mutant lineage spends in a single group in state $(s, i)$ in the asymptotic distribution of the mutant lineage. In sum, the basic reproductive number gives the expected number of successful emigrants produced by a lineage during its whole sojourn time in a single group and until its local extinction in that group, relative to the expected number of founders of such a lineage. Although the expression on the right-hand of eq. (12) does not appear previously in the literature, it pre- 
cisely corresponds to the mathematical definition of the basic reproductive number given in the literature (Caswell, 2000; Ellner and Rees, 2006, see Appendix D). Further, when there is only one demographic state, $R_{\mathrm{m}}(\tau, \theta)$ reduces to eq. (3) of Ajar (2003).

In Appendix $D$, we show that the basic reproductive number $R_{0}(\tau, \theta)$ predicts whether or not the mutant invades in the same way as the growth rate $\rho(\tau, \theta)$; namely

$$
R_{0}(\tau, \theta) \leq 1 \Longleftrightarrow \rho(\tau, \theta) \leq 1
$$

Hence, a strategy is uninvadable if the basic reproductive number is maximized. Suppose now that the number of founders $N_{\mathrm{F}}(\tau, \theta)$ is independent of the mutant; an example would be $N_{\mathrm{F}}(\tau, \theta)=1$ so there can be no propagule dispersal and individuals can only migrate independently of each others. Then, uninvadability can be characterized in terms of $R_{\mathrm{m}}(\tau, \theta)$ alone:

$$
R_{\mathrm{m}}(\tau, \theta) \leq 1 \Longleftrightarrow \rho(\tau, \theta) \leq 1
$$

Hence, a strategy is uninvadable if the expected number $R_{\mathrm{m}}(\tau, \theta)$ of successful emigrants is maximized.

Both reproductive numbers, $R_{0}$ and $R_{\mathrm{m}}$, count (emigrant) successful offspring as produced by a whole set of individuals in the lineage, and, by contrast to $W(\tau, \theta)$ and $W_{\mathrm{IF}}(\tau, \theta)$, are thus not individual-centered. In order to have a reproductive number that is expressed in terms of the personal fitness of a representative carrier of the mutant, we let the lineage fitness proxy of a mutant type $\tau$ in a resident $\theta$ population be given by

$$
R_{\mathrm{L}}(\tau, \theta)=\sum_{s^{\prime} \in S} \sum_{s \in S} \sum_{i \in I(s)} w\left(s^{\prime} \mid s, i\right) q_{\mathrm{L}}(s, i) .
$$

Here, $q_{\mathrm{L}}(s, i)$ is the probability that an individual randomly sampled from the mutant lineage over its lifetime in a single group finds itself in a group in state $(s, i)$ (see Table 1). This expression is a direct analogue to lineage fitness, with the only difference that the probability distribution $q_{\mathrm{L}}(s, i)$ depends on the lifetime of the lineage in a single group, and not on the asymptotic lineage distribution $u(s, i)$ as does lineage fitness. When there is only one demographic state, $R_{\mathrm{L}}(\tau, \theta)$ reduces to eq. (3) of Lehmann et al. (2015).

We show in Appendix $D$ that lineage fitness proxy $R_{\mathrm{L}}(\tau, \theta)$ predicts whether or not the mutant invades 
in the same way as the growth rate $\rho(\tau, \theta)$; that is,

$$
R_{\mathrm{L}}(\tau, \theta) \leq 1 \Longleftrightarrow \rho(\tau, \theta) \leq 1
$$

An uninvadable strategy thus also maximizes lineage fitness proxy.

\section{Results summary}

Summarizing all the above results, we have shown that the growth rate is equal to lineage fitness $\rho(\tau, \theta)=$ $W(\tau, \theta)$ and the following characterizations of the condition under which a mutant goes extinct are equivalent:

$$
W(\tau, \theta) \leq 1 \Longleftrightarrow W_{\mathrm{IF}}(\tau, \theta) \leq 1 \Longleftrightarrow R_{0}(\tau, \theta) \leq 1 \Longleftrightarrow R_{\mathrm{L}}(\tau, \theta) \leq 1 \underbrace{\Longleftrightarrow}_{\begin{array}{c}
N_{\mathrm{F}} \operatorname{does~not~} \\
\text { depend on } \tau
\end{array}} R_{\mathrm{m}}(\tau, \theta) \leq 1 .
$$

\section{Discussion}

Our results show that the different invasion fitness measures that have been proposed so far all equivalently determine which strategy is uninvadable, and that they can all be connected through their relationship to the growth rate of a mutant allele. The mathematical theory we present provides a formal framework for understanding the broad notion that different fitness measures must align (e.g., Metz et al., 1992; Roff, 2008; Akçay and Van Cleve, 2016). Our results also reveal interesting features of the different invasion fitness measures, which we now discuss.

\section{Lineage and inclusive fitness}

Uninvadability can be equivalently characterized in terms of lineage fitness or inclusive fitness. This duality is interesting as these two gene-centered invasion fitness measures are expressed in terms of different individual-centered fitness components experienced by representative carriers of the mutant allele. Lineage fitness is expressed only in terms of the personal fitness of a randomly drawn individual carrying the mutant allele, where the carrier is drawn from the distribution of group states experienced by members of the mutant lineage (all genetic-demographic-environmental states). In contrast, inclusive fitness is ex- 
pressed in terms of the direct fitness cost and relatedness weighted indirect fitness benefit accruing to a randomly drawn carrier of the mutant allele. Writing fitness in terms of cost and benefit requires making a comparison between the number of offspring produced by an individual expressing the mutant allele relative to expressing the resident allele. But in order for this comparison to be unbiased, how the fitness value of an offpsring depends on the demographic and/or environmental state in which it settles must be taken into account. Thus, each offspring needs to be appropriately weighted.

Importantly, we find that these weights are the neutral reproductive values of the monomorphic resident population regardless of the strength of selection on the mutant. The intuitive reason for this result is that reproductive value weighting "converts" number of offspring in different states into their proportionate contribution to the population. By choosing the conversion factors to be the neutral reproductive values of the resident allele, the inclusive fitness directly allows determining the increase (or decrease) in descendants into the far future that a typical carrier of the mutant allele leaves relative to the typical carrier of the resident allele, in a monomorphic resident population. This result is consistent with previous population genetic formulations of allele frequency change in class-structured populations under arbitrary strength of selection (Lehmann and Rousset, 2014). Our analysis thus generalizes the exact version of inclusive fitness (e.g., Queller, 1992; Frank, 1997; Gardner et al., 2011) to class-structured populations with variable number of interaction partners, and shows that the standard neutral reproductive value weighting (e.g., Taylor and Frank, 1996; Rousset, 2004) is maintained in this generalization.

Inclusive fitness makes explicit that the force of selection on a mutant allele depends on (i) how individuals in different demographic and environmental states contribute differently to the gene pool and on (ii) the genetic association between individuals due to local common ancestry, regardless of the complexity of the biological situation at hand and the strength of selection. These biological features, hidden in the other invasion fitness measures, also become apparent if one considers only the first-order effects of selection on the growth rate when the evolving traits have continuous values. This is the situation usually considered in the adaptive dynamics and inclusive fitness literature where one looks for evolutionary attractors (Taylor, 1996; Geritz et al., 1998; Rousset, 2004; Dercole and Rinaldi, 2008). In this situation, the sensitivity of the growth rate with respect to changes in trait value boils down to the inclusive fitness effect derived previously by the direct fitness method (Taylor and Frank, 1996; Rousset, 2004, see Box 2 and Appendix E.2 for this connection). Hence, our model makes explicit that the direct fitness method amounts to computing the sensitivity of the growth rate of the mutant with respect to changes in mutant strategy under a general 
class structure and with environmental heterogeneity (see also Rousset, 2004, pp. 194-196 for a conjecture on that point).

Our analysis thus demonstrates connections between the various theoretical approaches for characterizing adaptations in heterogeneous populations. But depending on the type of questions and insight desired, either inclusive or lineage fitness formulations might be better suited. For instance, lineage fitness may be easier to measure, as it only relies on measuring personal fitness of a representative sample of individuals of the mutant type (see Akçay and Van Cleve, 2016 for further discussions on using invasion fitness measures for empirical system).

\section{Reproductive numbers}

We also derived an explicit expression for the basic reproductive number, $R_{0}$, for a group-structured population, which was shown to depend on the ratio of the total lifetime number $R_{\mathrm{m}}$ of successful emigrants produced by a typical group colonized by members of the mutant lineage, to the expected number $N_{\mathrm{F}}$ of colonizers of such a typical group. The basic reproductive number is the usual invasion fitness proxy in evolutionary biology and epidemiology (Caswell, 2000; Ellner and Rees, 2006) and is usually used as it simplifies the characterization of the condition under which a mutant invades. It circumvents the need to compute explicitly the growth rate $\rho$, (the eigenvalue of the transition matrix $\mathbf{A}$ ), and only requires a matrix inversion (see Appendix D). When individuals disperse independently and not in clusters (i.e., no propagule dispersal), the basic reproductive number reduces to the number of successful emigrants $R_{\mathrm{m}}$. Mathematically however, our expression for $R_{\mathrm{m}}$ (eq. (12)) differs from the expression of $R_{\mathrm{m}}$ initially introduced as a measure of invasion fitness by Metz and Gyllenberg (2001), insofar as the frequency distribution of the group states of a typically colonized group may depend on the mutant type, which is consistent with the formal proof of $R_{\mathrm{m}}$ derived by Massol et al. (2009).

Two further points are worth mentioning concerning the reproductive numbers, $R_{0}$ and $R_{\mathrm{m}}$. First, while no relatedness appears explicitly in them, they take inclusive fitness effects into account in the same amount as inclusive fitness (or lineage fitness) does. Second, the reproductive numbers count successful emigrant offspring produced by a whole set of individuals, and thus do not give net successful offspring produced by a representative carrier of the mutant allele. In order to have a fully individual-centered measure of invasion fitness, which keeps the attractive computational features of the reproductive numbers, 
we derived an expression for lineage fitness proxy $R_{\mathrm{L}}$. This is the personal fitness of a mutant lineage member randomly sampled from the distribution quantifying the lifetime of the mutant lineage in a local group. This allows one to determine uninvadability with the same generality and simplicity as $R_{0}$, but with the same biological interpretation as lineage fitness.

\section{Generalizations}

To obtain our results, we assumed a population of infinite size but allowed for limited dispersal between any local group and local demographic and/or environmental state. This allows one to describe, in at least a qualitative way, different metapopulation processes as well as group (or propagule) reproduction processes subject to local demographic and environmental stochasticity. Conceptually, our qualitative results concerning the generic form of the fitness measures should carry over to isolation-by-distance models and finite total population size once the growth rate is interpreted as the fixation probability.

We also only considered halploid reproduction, but diploid reproduction would not produce qualitatively different results concerning the expressions of lineage fitness, inclusive fitness, or the reproductive numbers. In the case of diploidy, one needs to add an additional class structure within each demographic state so that individuals are either homozygous or heterozygous and produce these two types of offspring. The same extension is needed for class structure such as age or stage (see Box 3 and Appendix $\mathrm{E}$ for an example involving stage structure). An extension to continuous classes is also straightforward as its suffices to replaces eigenvectors by eigenfunctions in the characterization of the growth rate (Harris, 1963), and all other calculations should carry over conceptually unchanged (but replacing sums by appropriate integrals). Our approach, however, breaks down when there are global environmental fluctuations affecting all groups in the population simultaneously, in which case the stochastic growth rate needs to be used to ascertain uninvadability (Svardal et al., 2015). Hence, a completely general interpretation of the growth rate of a mutant in terms of individual-centered fitness components, covering all possible biological heterogeneities, is still lacking. But for local heterogeneities, there is a generality and consistency in the interpretation of the force of a selection on a mutant allele that befits the generality of natural selection. 


\section{References}

Ajar, E. 2003. Analysis of disruptive selection in subdivided populations. BMC Evolutionary Biology 3:22.

Akçay, E. and J. Van Cleve. 2012. Behavioral responses in structured populations pave the way to group optimality. American Naturalist 179:257-269.

Akçay, E. and J. Van Cleve. 2016. There is no fitness but fitness and the lineage is its bearer. Philosophical transactions of the Royal Society B 371:20150085.

Avila, P. and L. Fromhage. 2015. No synergy needed: ecological constraints favor the evolution of eusociality. American Naturalist 186.

Bulmer, M. G. 1986. Sex ratio theory in geographically structured populations. Heredity 56:69-73.

Cadet, C., R. Ferrière, J. A. J. Metz, and M. van Baalen. 2003. The evolution of dispersal under demographic stochasticity. Am Nat 162:427-41.

Caswell, H. 2000. Matrix Population Models. Sinauer Associates, Massachusetts.

Charlesworth, B. 1994. Evolution in Age-Structured Populations. Cambridge University Press, Cambridge, 2th edn.

Chesson, P. L. 1981. Models for spatially distributed populations: the effect of within-patch variability. Theoretical Population Biology 19:288-325.

Chesson, P. L. 1984. Persistence of a Markovian population in a patchy environment. Zeitschrift für Wahrscheinlichkeitstheorie 66:97-107.

Cohen, J. E. 1979. Long-run growth rates of discrete multiplicative processes in markovian environments. Journal of Mathematical Analysis and Applications 69:243-251.

Dawkins, R. 1978. Replicator selection and the extended phenotype. Zeitschrift für Tierpsychologie 47:6176.

Day, T. 2001. Population structure inhibits evolutionary diversification under competition for resources.

Genetica 112:71-86. 
Dercole, F. and S. Rinaldi. 2008. Analysis of Evolutionary Processes: The Adaptive Dynamics Approach and Its Applications. Princeton University Press, Princeton, NJ.

Ellner, S. P. and M. Rees. 2006. Integral projection models for species with complex demography. Am Nat 167:410-28.

Eshel, I. 1991. Game theory and population dynamics in complex genetical systems: the role of sex in short term and in long term evolution. In Selten, R. (ed.), Game equilibrium models I, pp. 6-28. Springer.

Eshel, I. 1996. On the changing concept of evolutionary population stability as a reflection of a changing point of view in the quantitative theory of evolution. Journal of Mathematical Biology 34:485-510.

Eshel, I. and M. W. Feldman. 1984. Initial increase of new mutants and some continuity properties of ESS in two-locus systems. The American Naturalist 124:631-640.

Ewens, W. J. 2004. Mathematical Population Genetics. Springer-Verlag, New York.

Ferrière, R. and M. Gatto. 1995. Lyapunov exponents and the mathematics of invasion in oscillatory or chaotic populations. Theoretical Population Biology 48:126-171.

Frank, S. A. 1997. The Price equation, Fisher's fundamental theorem, kin selection, and causal analysis. Evolution 51:1712-1729.

Frank, S. A. 1998. Foundations of Social Evolution. Princeton University Press, Princeton, NJ.

Gandon, S. 1999. Kin competition, the cost of inbreeding and the evolution of dispersal. Journal of Theoretical Biology 200:345-364.

Gardner, A., S. A. West, and G. Wild. 2011. The genetical theory of kin selection. Journal of Evolutionary Biology 24:1020-1043.

Geritz, S. A. H., E. Kisdi, G. Meszéna, and J. A. J. Metz. 1998. Evolutionarily singular strategies and the adaptive growth and branching of the evolutionary tree. Evolutionary Ecology 12:35-57.

Grey, D., V. Hutson, and E. Szathmary. 1995. A re-examination of the stochastic corrector model. Proceedings of the Royal Society B: Biological Sciences 262:29-35. 
Grinstead, C. M. and J. L. Snell. 1997. Introduction to Probability. American Mathematical Society, Providence, RI, second edn.

Haig, D. 1997. Parental antagonism, relatedness asymmetries, and genomic imprinting. Proceedings of the Royal Society of London Series B-Biological Sciences 264:1657-1662.

Hammerstein, P. 1996. Darwinian adaptation, population genetics and the streetcar theory of evolution. Journal of Mathematical Biology 34:511-532.

Harris, T. E. 1963. The Theory of Branching Processes. Springer, Berlin.

Iosifescu, M. 2007. Finite Markov Processes and Their Applications. Dover, New York.

Johnstone, R. A. and M. A. Cant. 2010. The evolution of menopause in cetaceans and humans: the role of demography. Proceedings of the Royal Society B-Biological Sciences 277:3765-71.

Karlin, S. and H. M. Taylor. 1975. A First Course in Stochastic Processes. Academic Press, San Diego.

Lehmann, L. 2008. The adaptive dynamics of niche constructing traits in spatially subdivided populations: evolving posthumous extended phenotypes. Evolution 62:549-566.

Lehmann, L., I. Alger, and J. W. Weibull. 2015. Does evolution lead to maximizing behavior? Evolution 69:1858-1873.

Lehmann, L. and F. Rousset. 2010. How life-history and demography promote or inhibit the evolution of helping behaviors. Philosophical transactions of the Royal Society B 365:2599-2617.

Lehmann, L. and F. Rousset. 2014. The genetical theory of social behaviors. Philosophical transactions of the Royal Society B 369:1-18.

Lion, S. 2013. Multiple infections, kin selection and the evolutionary epidemiology of parasite traits. Journal of Evolutionary Biology 26:2107-2122.

Lion, S. and S. Gandon. 2009. Habitat saturation and the spatial evolutionary ecology of altruism. Journal of Evolutionary Biology 22:1487-1502.

Lion, S. and M. van Baalen. 2007. Self-structuring in spatial evolutionary ecology. Ecology Letters 11:277295. 
Massol, F., V. Calcagno, and J. Massol. 2009. The metapopulation fitness criterion: proof and perspectives.

Theoretical Population Biology 75:183-200.

Maynard Smith, J. 1982. Evolution and the Theory of Games. Cambridge University Press, Cambridge.

McNamara, J., A. I. Houston, and E. J. Collins. 2001. Optimality models in Behavioral Ecology. SIAM Review 43:413-466.

Metz, J. and M. Gyllenberg. 2001. How should we define fitness in structured metapopulation models? including an application to the calculation of evolutionary stable dispersal strategies. Proceedings of the Royal Society of London Series B-Biological Sciences 268:499-508.

Metz, J. A. J. 2011. Thoughts on the geometry of meso-evolution: collecting mathematical elements for a post-modern synthesis. In Chalub, F. A. C. C. and J. Rodrigues (eds.), The mathematics of Darwin's legacy, Mathematics and biosciences in interaction. Birkhäuser, Basel.

Metz, J. A. J., S. A. H. Geritz, G. Meszéna, F. J. A. Jacobs, and J. S. van Heerwaarden. 1996. Adaptive dynamics: a geometrical study of the consequences of nearly faithful reproduction. In van Strien, S. J. and S. M. Verduyn Lunel (eds.), Stochastic and Spatial Structures of Dynamical Systems, pp. 183-231. North-Holland, Amsterdam.

Metz, J. A. J., R. M. Nisbet, and S. A. H. Geritz. 1992. How should we define fitness for general ecologica scenarios? Trends in Ecology and Evolution 7:198-202.

Moran, P. A. P. 1964. On the nonexistence of adaptive topographies. Annals of Human Genetics 27:383-393.

Motro, U. 1982. Optimal rates of dispersal I. Haploid populations. Theoretical Population Biology 21:394411.

Mullon, C., K. L., and L. Lehmann. 2016. Evolutionary stability of multiple traits in subdivided populations. American Naturalist In revision.

Mullon, C. and L. Lehmann. 2014. The robustness of the weak selection approximation for the evolution of altruism against strong selection. Journal of Evolutionary Biology 27:2272-2282.

Parker, G. A. and J. Maynard Smith. 1990. Optimality theory in evolutionary biology. Science 349:27-33. 
Pen, I. 2000. Reproductive effort in viscous populations. Evolution 54:293-297.

Queller, D. C. 1992. A general model for kin selection. Evolution 46:376-380.

Rand, D. A., W. H. B., and M. J. M. 1994. Dynamics and evolution: evolutionarily stable attractors, invasion exponents and phenotype dynamics. Philosophical Transactions of the Royal Society B-Biological Sciences 343:261-83.

Reeve, H. K. and P. W. Sherman. 1993. Adaptation and the goal of evolutionary research. The Quarterly Review of Biology 68:1-32.

Roff, D. A. 2008. Defining fitness in evolutionary models. Journal of Genetics 87:339-348.

Ronce, O. and D. Promislow. 2010. Kin competition, natal dispersal and the moulding of senescence by natural selection. Proc Biol Sci 277:3659-67.

Rousset, F. 2004. Genetic Structure and Selection in Subdivided Populations. Princeton University Press, Princeton, NJ.

Rousset, F. and O. Ronce. 2004. Inclusive fitness for traits affecting metapopulation demography. Theoretical Population Biology 65:127-141.

Svardal, H., C. Rueffler, and J. Hermisson. 2015. A general condition for adaptive genetic polymorphism in temporally and spatially heterogeneous environments. Theoretical Population Biology 99:76-97.

Szathmary, E. and L. Demeter. 1987. Group Selection of Early Replicators and the Origin of Life. Journal of Theoretical Biology 128:463-486.

Taylor, P. D. 1988. An inclusive fitness model for dispersal of offspring. Journal of Theoretical Biology $130: 363-378$.

Taylor, P. D. 1996. Inclusive fitness arguments in genetic models of behaviour. Journal of Mathematical Biology 34:654-674.

Taylor, P. D. and S. A. Frank. 1996. How to make a kin selection model. Journal of Theoretical Biology 180:27-37. 
Taylor, P. D. and A. J. Irwin. 2000. Overlapping generations can promote altruistic behavior. Evolution 54:1135-1141.

Thieme, H. R. 2009. Spectral bound and reproduction number for infinite-dimensional population structure and time heterogeneity. SIAM Journal of applied mathematics 70:188-211.

Tuljapurkar, S. 1989. An uncertain life: demography in random environments. Theoretical Population Biology 35:227-94.

Tuljapurkar, S., C. C. Horvitz, and J. B. Pascarella. 2003. The many growth rates and elasticities of populations in random environments. Am. Nat. 162:489-502.

van Baalen, M. 2013. The unit of adaptation, the emergence of individuality, and the loss of evolutionary sovereignty. In Huneman, P. and F. Bouchard (eds.), From Groups to Individuals. Evolution and Emerging Individuality, pp. 117-140. MIT Press.

van Baalen, M. and A. Rand. 1998. The unit of selection in viscous populations and the evolution of altruism. Journal of Theoretical Biology 193:631-648.

Van Cleve, J. 2015. Social evolution and genetic interactions in the short and long term. Theoretical Population Biology 103:2-26.

Weissing, F. J. 1996. Genetic versus phenotypic models of selection: can genetics be neglected in a longterm perspective? Journal of Mathematical Biology 34:533-555.

Wenseleers, T., A. Gardner, and K. R. Foster. 2010. Social evolution theory: a review of methods and approaches. In Szekely, T., A. Moore, and J. Komdeur (eds.), Social Behaviour: Genes, Ecology and Evolution, pp. 132-158. Cambridge University Press, Cambridge.

Wild, G. 2011. Inclusive fitness from multitype branching processes. Bull. Math. Biol. 73:1028-51.

Wild, G., A. Gardner, and S. A. West. 2009. Adaptation and the evolution of parasite virulence in a connected world. Nature 459:983-986.

Wright, S. 1931. Evolution in Mendelian populations. Genetics 16:97-159. 


\begin{tabular}{|c|c|}
\hline Function & Definition \\
\hline$a\left(s^{\prime}, i^{\prime} \mid s, i\right)$ & Element $\left(s^{\prime}, i^{\prime} ; s, i\right)$ of the matrix $\mathbf{A}=\mathbf{P}+\mathrm{D}$. \\
\hline$p\left(s^{\prime}, i^{\prime} \mid s, i\right)$ & Element $\left(s^{\prime}, i^{\prime} ; s, i\right)$ of the matrix $\mathbf{P}$. \\
\hline$d\left(s^{\prime}, i^{\prime} \mid s, i\right)$ & Element $\left(s^{\prime}, i^{\prime} ; s, i\right)$ of the matrix $\mathbf{D}$. \\
\hline$w\left(s^{\prime} \mid s, i\right)=\frac{1}{i} \sum_{i^{\prime} \in I\left(s^{\prime}\right)} i^{\prime} a\left(s^{\prime}, i^{\prime} \mid s, i\right)$ & $\begin{array}{l}\text { Expected number of successful offspring, which settle in groups of type } \\
s^{\prime} \text {, and are produced by a single mutant individual given that it resides in } \\
\text { a group in state } s \text { and when there are } i \text { mutants. }\end{array}$ \\
\hline$w_{\mathrm{p}}\left(s^{\prime} \mid s, i\right)=\frac{1}{i} \sum_{i^{\prime} \in I\left(s^{\prime}\right)} i^{\prime} p\left(s^{\prime}, i^{\prime} \mid s, i\right)$ & $\begin{array}{l}\text { Expected number of philopatric offspring, which settle in groups of type } \\
s^{\prime} \text {, and are produced by a single mutant individual given that it resides in } \\
\text { a group in state } s \text { and when there are } i \text { mutants. }\end{array}$ \\
\hline$w_{\mathrm{d}}\left(s^{\prime} \mid s, i\right)=\frac{1}{i} \sum_{i^{\prime} \in I\left(s^{\prime}\right)} i^{\prime} d\left(s^{\prime}, i^{\prime} \mid s, i\right)$ & $\begin{array}{l}\text { Expected number of successful dipsersing offspring, which settle in } \\
\text { groups of type } s^{\prime} \text {, and are produced by a single mutant individual given } \\
\text { that it resides in a group in state } s \text { and when there are } i \text { mutants. }\end{array}$ \\
\hline$u(s, i)$ & $\begin{array}{l}\text { Asymptotic probability that a mutant lineage finds itself in a group in } \\
\text { state }(s, i) \text {. This is element }(s, i) \text { of the right eigenvector } \mathbf{u} \text { of } \mathrm{A} \text { associated } \\
\text { to its leading positive eigenvalue } \rho \text {; namely, } \rho \mathbf{u}=\mathrm{Au} \text {. }\end{array}$ \\
\hline$q(s)=\frac{\sum_{i \in I(s)} i u(s, i)}{\sum_{s \in S} \sum_{i \in I(s)} i u(s, i)}$ & $\begin{array}{l}\text { Asymptotic probability that a randomly drawn mutant lineage member } \\
\text { find itself in a group in state } s \text {. }\end{array}$ \\
\hline$q(i \mid s)=\frac{i u(s, i)}{\sum_{i \in I(s)} i u(s, i)}$ & $\begin{array}{l}\text { Asymptotic probability that, conditional on being sampled in a group in } \\
\text { state } s \text {, a randomly sampled mutant individual from the mutant lineage } \\
\text { has } i-1 \text { mutant neighbors. }\end{array}$ \\
\hline$r(s)=\sum_{i \in I(s)} \frac{(i-1)}{(n(s)-1)} q(i \mid s)$ & $\begin{array}{l}\text { Asymptotic probability that, conditional on being sampled in a group in } \\
\text { state } s \text {, an individual carrying the mutant experiences a randomly } \\
\text { sampled neighbour that also carries the mutant allele. This is a measure } \\
\text { of pairwise relatedness between individuals in a group. }\end{array}$ \\
\hline$w^{\circ}\left(s^{\prime} \mid s\right)$ & $\begin{array}{l}\text { Expected number of successful offspring, which settle in groups of type } \\
s^{\prime} \text {, and are produced by a single mutant individual residing in a group in } \\
\text { state } s \text { in a monomorphic resident population. }\end{array}$ \\
\hline$v^{\circ}(s)=\sum_{s^{\prime} \in S} v^{\circ}\left(s^{\prime}\right) w^{\circ}\left(s^{\prime} \mid s\right)$ & $\begin{array}{l}\text { Reproductive value of a single individual reproducing in a group in state } \\
s \text { in a monomorphic resident population. }\end{array}$ \\
\hline
\end{tabular}

Table 1: Definitions of the functions and vectors used for lineage fitness, inclusive fitness, and the reproductive number. 


\begin{tabular}{ll}
\hline Function & Definition \\
\hline$u_{0}(s, i)$ & $\begin{array}{l}\text { Asymptotic probability that a group initiated by a local lineage starts } \\
\text { in state }(s, i) . \text { This is element }(s, i) \text { of the right eigenvector } \mathbf{u}_{0} \text { of }\end{array}$ \\
& $R_{0} \mathbf{u}_{0}=\mathbf{R} \mathbf{u}_{0}$ where $\mathbf{R}=\mathbf{D}(\mathbf{I}-\mathbf{P})^{-1}$ is the next generation matrix. \\
& $\begin{array}{l}\text { Expected number of demographic times steps the mutant lineage } \\
\text { spends in state }\left(s^{\prime}, i^{\prime}\right) \text { over its lifetime in a single group given that the }\end{array}$ \\
& group started in state $(s, i)$. This is element $\left(s^{\prime}, i^{\prime} ; s, i\right)$ of the matrix \\
& $(\mathbf{I}-\mathbf{P})^{-1}$ of sojourn times. \\
& $\begin{array}{l}\text { Average of the expected amount of time the mutant lineage spends in } \\
\text { state }\left(s^{\prime}, i^{\prime}\right) \text { over its lifetime in a single group. }\end{array}$ \\
$N_{\mathrm{F}}(\tau, \theta)=\sum_{s \in S} \sum_{i \in I(s)} i u_{0}(s, i)$ & Expected number of founders in a single group of the mutant lineage. \\
$N_{\mathrm{L}}(\tau, \theta)=\sum_{s \in S} \sum_{i \in I(\zeta)} i \bar{t}(s, i)$ & $\begin{array}{l}\text { Average total size of the mutant lineage over its lifetime in a single } \\
\text { group. }\end{array}$ \\
& $\begin{array}{l}\text { Probability that an individual randomly sampled from the mutant } \\
\text { lineage over its lifetime in a single group finds itself in a group in } \\
\text { state }(s, i) .\end{array}$ \\
\hline$q_{\mathrm{L}}(s, i)=\frac{i t(s, i)}{N_{\mathrm{L}}(\tau, \theta)}$ &
\end{tabular}

Continuation of Table 1. 
Box I. Weighted least square regression. We here show how to obtain the $\operatorname{cost} \gamma\left(s^{\prime} \mid s\right)$ and benefit $\beta\left(s^{\prime} \mid s\right)$ in eq. (8). These are found by minimizing for each state $s \in \mathcal{S}$ the sum of squared errors $\epsilon_{i}$ weighted by the probabilities $q(i \mid s)$ :

$$
Q(\gamma, \beta)=\sum_{i \in I(s)} \epsilon_{i}^{2} q(i \mid s)
$$

That is, from eq. (8), we minimize

$$
Q(\gamma, \beta)=\sum_{i \in I(s)}\left[w\left(s^{\prime} \mid s, i\right)-\left(w^{\circ}\left(s^{\prime} \mid s\right)-\gamma\left(s^{\prime} \mid s\right)+\beta\left(s^{\prime} \mid s\right) \frac{(i-1)}{n(s)-1}\right)\right]^{2} q(i \mid s),
$$

with respect to $\gamma$ and $\beta$. From the prediction theorem for minimum square error prediction (Karlin and Taylor, 1975, p. 465), we then have $\sum_{i \in I(s)} \epsilon_{i} q(i \mid s)=0$ for all $s \in \mathcal{S}$, which is one of the main feature we use to obtain the expression for inclusive fitness (see Appendix (). 
Box II. Sensitivity of the growth rate. We here provide an expression for the sensitivity of the growth rate when the mutant trait value is varied; that is, the derivative of the growth rate when $\Theta=\mathbb{R}$, which is sufficient to evaluate singular strategies and convergence stable states (Taylor, 1996; Rousset, 2004). In Appendix E.2, we prove that the sensitivity of the growth rate is

$$
\frac{\partial \rho(\tau, \theta)}{\partial \tau}=\sum_{s^{\prime} \in S} \sum_{s \in S} v^{\circ}\left(s^{\prime}\right)\left[\frac{\partial w\left(s^{\prime} \mid s, \tau_{j}, \tau_{-j}\right)}{\partial \tau_{j}}+(n(s)-1) \frac{\partial w\left(s^{\prime} \mid s, \tau_{j}, \tau_{-j}\right)}{\partial \tau_{k}} r^{\circ}(s)\right] q^{\circ}(s)
$$

where $w\left(s^{\prime} \mid s, \tau_{j}, \tau_{-j}\right)$ is the personal fitness of an individual with phenotype $\tau_{j}$, when its group members have phenotype profile $\tau_{-j}=\left(\tau_{1}, \ldots, \tau_{j-1}, \tau_{j+1}, \ldots \tau_{n(s)-1}\right)$, which is the vector collecting the phenotypes of the $n(s)-1$ neighbors of an individual $j$ and $k \neq j$, and all derivatives are evaluated at the resident values $\theta$. Note that here, both the probability $q^{\circ}(s)$ that a mutant experiences a group in state $s$ and relatedness $r^{\circ}(s)$ are evaluated in a monomorphic resident population (neutral process). Given further specific biological assumptions on the underlying demographic process, we then recover from the above derivative the expression for the inclusive fitness effect derived by the direct fitness method for the island model (Taylor and Frank, 1996; Rousset and Ronce, 2004, see Appendix E.3.1). 


\section{Box III. Lineage and inclusive fitness for class-structure under fixed demography.}

Suppose that each group is of constant size but that each individual within a group can belong to one of $n_{\mathrm{c}}$ classes where the set of classes is $C=\left\{1, \ldots, n_{\mathrm{c}}\right\}$. An example would be age structure due to overlapping generations or different castes of social insects like workers and queens. For such a class structured

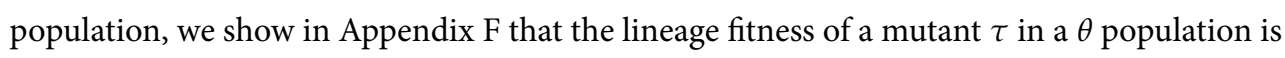

$$
W(\tau, \theta)=\sum_{\mathbf{i} \in I} \sum_{y \in C} \sum_{x \in C} w(y, x, \mathbf{i}) q(x, \mathbf{i})
$$

where $w(y, x, \mathbf{i})$ is the expected number of class $y$ offspring produced by a class $x$ mutant when in a group in state $\mathbf{i}=\left(i_{1}, \ldots, i_{n_{\mathrm{c}}}\right) \in I$, which is the vector of the number of mutant alleles in class 1 to $n_{\mathrm{c}}$. Here, $I=\left(I_{1} \times \cdots \times I_{n_{c}}\right)$ is the set of possible group states with $I_{x}=\left\{0,1, \ldots, n_{x}\right\}$ being the set of the number of mutant alleles in class $x$ and $n_{x}$ is the number of individuals in that class. In complete analogy with the demographically structured population case, $q(x, \mathbf{i})$ is the probability that a randomly sampled lineage member finds itself in class $x$ and its group state is $i$. The inclusive fitness expression for this model is

$$
W_{\mathrm{IF}}(\tau, \theta)=1+\sum_{y \in C} \sum_{x \in C} v^{\circ}(y)\left[-\gamma(y, x)+\sum_{z \in C} \beta_{z}(y, x) r(z \mid x)\right] q(x),
$$

where $q(x)$ is the probability that a randomly sampled individual from the mutant lineage finds itself in class $x, \gamma(y, x)$ is the additive effect on the number of class $y$ offspring produced by a class $x$ individual when expressing the mutant instead of the resident allele, $\beta_{z}(y, x)$ is the additive effect on this fitness stemming from group neighbors in class $z$ expressing the mutant instead of the resident allele, and $r(z \mid x)$ is the probability that, conditional on being sampled in class $x$, an individual carrying the mutant experiences a randomly sampled neighbour in class $z$ that also carries the mutant allele. 


\section{Appendix A: Properties of the monomorphic resident population}

The demographic equilibrium for a monomorphic resident $\tau$ population described in the main text can be expressed as

$$
p^{\circ}\left(s^{\prime}\right)=\sum_{s \in \mathcal{S}} p^{\circ}\left(s^{\prime} \mid s\right) p^{\circ}(s)
$$

where $p^{\circ}(s)$ is the neutral stationary probability that in group is in state $s$ and $p^{\circ}\left(s^{\prime} \mid s\right)$ denotes the neutral transition probability from state $s$ to $s^{\prime}$ (possibly depending endogenously on the distribution $p^{\circ}(s)$ ).

We now prove that in a monomorphic $\theta$ population the neutral transition matrix $\mathrm{A}^{\circ}$ has dominant eigenvalue $\rho(\theta, \theta)=1$. We do so by constructing a positive left eigenvector $\mathbf{v}^{\circ}>0$ of $\mathbf{A}^{\circ}$ with unit eigenvalue (i.e., such that $\mathbf{v}^{\circ} \mathbf{A}^{\circ}=\mathbf{v}^{\circ}$ ). Then, since $\mathbf{A}^{\circ}$ is irreducible and non-negative $\left(\right.$ and $\mathbf{v}^{\circ}>0$ ), the Perron-Frobenius theorem tells us that the dominant eigenvalue of $\mathbf{A}^{\circ}$ is one (e.g., Karlin and Taylor, 1975). We construct $\mathbf{v}^{\circ}=\left(v^{\circ}(1,1), \ldots, v^{\circ}(1, n(1)), v^{\circ}(1,2), \ldots\right)$ with $(s, i)$ element

$$
v^{\circ}(s, i)=v^{\circ}(s) i,
$$

where $v^{\circ}(s)>0$ corresponds to the reproductive value of an individual in class $s$ (see Taylor, 1996 and Rousset, 2004). By definition, reproductive values satisfy

$$
v^{\circ}(s)=\sum_{s^{\prime} \in S} v^{\circ}\left(s^{\prime}\right) w^{\circ}\left(s^{\prime} \mid s\right)
$$

To show that our construction of $\mathbf{v}^{\circ}$ is a left eigenvector of $\mathbf{A}^{\circ}$, we first write the $(s, i)$ element of $\mathbf{v}^{\circ} \mathbf{A}^{\circ}$ by using eq. A.2 as

$$
\sum_{s^{\prime} \in \mathcal{S}} \sum_{i^{\prime} \in I\left(s^{\prime}\right)} v^{\circ}\left(s^{\prime}, i^{\prime}\right) a^{\circ}\left(s^{\prime}, i^{\prime} \mid s, i\right)=\sum_{s^{\prime} \in S} \sum_{i^{\prime} \in I\left(s^{\prime}\right)} v^{\circ}\left(s^{\prime}\right) i^{\prime} a^{\circ}\left(s^{\prime}, i^{\prime} \mid s, i\right) .
$$

Then, we note that the total expected number of mutant individuals in a group of type $s^{\prime}$ produced by a group of type $(s, i)$ can be written in two ways,

$$
\sum_{i^{\prime} \in I\left(s^{\prime}\right)} i^{\prime} a^{\circ}\left(s^{\prime}, i^{\prime} \mid s, i\right)=w^{\circ}\left(s^{\prime} \mid s\right) i
$$


where, owing to neutrality, fitness $w^{\circ}\left(s^{\prime} \mid s\right)$ is independent of $i$. Using eq. (A.5) first and (A.3) second, the $(s, i)$ element of $\mathbf{v}^{\circ} \mathbf{A}^{\circ}$ can thus be written as

$$
\begin{aligned}
\sum_{s^{\prime} \in \mathcal{S}} \sum_{i^{\prime} \in I\left(s^{\prime}\right)} v^{\circ}\left(s^{\prime}\right) i^{\prime} a^{\circ}\left(s^{\prime}, i^{\prime} \mid s, i\right) & =\sum_{s^{\prime} \in \mathcal{S}} v^{\circ}\left(s^{\prime}\right) w^{\circ}\left(s^{\prime} \mid s\right) i \\
& =v^{\circ}(s) i \\
& =v^{\circ}(s, i)
\end{aligned}
$$

i.e., as the $(s, i)$ element of $\mathbf{v}^{\circ}$, which shows that our construction of $\mathbf{v}^{\circ}$ is indeed a left eigenvector of $\mathbf{A}^{\circ}$ with unit eigenvalue, as required.

\section{Appendix B: Lineage fitness}

We here prove that $\rho(\tau, \theta)=W(\tau, \theta)$ (eq. (可) of the main text). To that aim, we first note that eq. (A.5) holds out of neutrality and that

$$
\sum_{i^{\prime} \in I\left(s^{\prime}\right)} i^{\prime} a\left(s^{\prime}, i^{\prime} \mid s, i\right)=w\left(s^{\prime} \mid s, i\right) i
$$

since the right hand side is the total expected number of mutant individuals in a group of type $s^{\prime}$ produced by a group of type $(s, i)$. Second, we let $\mathbf{n}=(1,2, \ldots, n(1), 1,2 \ldots, n(2), \ldots, n(s))$ and premultiply $\rho \mathbf{u}=\mathbf{A u}$ by $\mathbf{n}$ gives $\mathbf{n} \cdot \rho \mathbf{u}=(\mathbf{n} \cdot \mathbf{A u})$, where $\cdot$ is the dot product. Using eq. (B.1) we have

$$
\begin{aligned}
\rho(\tau, \theta) & =\frac{1}{\mathbf{n} \cdot \mathbf{u}}(\mathbf{n} \cdot \mathbf{A u}) \\
& =\frac{1}{\mathbf{n} \cdot \mathbf{u}} \sum_{s^{\prime} \in S} \sum_{i^{\prime} \in I\left(s^{\prime}\right)} \sum_{s \in S} \sum_{i \in I(s)} i^{\prime} a\left(s^{\prime}, i^{\prime} \mid s, i\right) u(s, i) \\
& =\frac{1}{\mathbf{n} \cdot \mathbf{u}} \sum_{s^{\prime} \in S} \sum_{s \in S} \sum_{i \in I(s)} w\left(s^{\prime} \mid s, i\right) i u(s, i) .
\end{aligned}
$$

Using the definitions of $q(i \mid s)$ and $q(s)$ given in the Table 1 of the main text (where $\sum_{s \in S} q(s)=1$ and $\left.\sum_{i \in I(s)} q(i \mid s)=1\right)$, we can then write

$$
\rho(\tau, \theta)=\sum_{s^{\prime} \in \mathcal{S}} \sum_{s \in \mathcal{S}} \sum_{i \in I(s)} w\left(s^{\prime} \mid s, i\right) q(i \mid s) q(s) .
$$


The right hand side is exactly $W(\tau, \theta)$, whereby $\rho(\tau, \theta)=W(\tau, \theta)$.

\section{Appendix C: Inclusive fitness}

Here, we prove that the uninvadability condition can be expressed in terms of inclusive fitness (eq. (10)).

For this, we premultiply $\rho \mathbf{u}=\mathbf{A} \mathbf{u}$ by $\mathbf{v}^{\circ}$, which gives $\mathbf{v}^{\circ} \cdot \rho \mathbf{u}=\left(\mathbf{v}^{\circ} \cdot \mathbf{A u}\right)$. Using eq. (A.2) then entails

$$
\begin{aligned}
\rho(\tau, \theta) & =\frac{1}{\mathbf{v}^{\circ} \cdot \mathbf{u}} \sum_{s^{\prime} \in S} \sum_{i^{\prime} \in I\left(s^{\prime}\right)} \sum_{s \in S} \sum_{i \in I(s)} v^{\circ}\left(s^{\prime}\right) i^{\prime} a\left(s^{\prime}, i^{\prime} \mid s, i\right) u(s, i) \\
& =\frac{1}{\mathbf{v}^{\circ} \cdot \mathbf{u}} \sum_{s^{\prime} \in S} \sum_{s \in S} \sum_{i \in I(s)} v^{\circ}\left(s^{\prime}\right) w\left(s^{\prime} \mid s, i\right) i u(s, i)
\end{aligned}
$$

and using

$$
V_{\mathrm{T}}=\frac{\mathbf{v}^{\circ} \cdot \mathbf{u}}{\mathbf{n} \cdot \mathbf{u}}=\sum_{s \in \mathcal{S}} v^{\circ}(s) q(s)
$$

which is the average reproductive value, yields

$$
\rho(\tau, \theta)=\frac{1}{V_{\mathrm{T}}} \sum_{s^{\prime} \in S} \sum_{s \in S} \sum_{i \in I(s)} v^{\circ}\left(s^{\prime}\right) w\left(s^{\prime} \mid s, i\right) \frac{i u(s, i)}{\mathbf{n} \cdot \mathbf{u}}
$$

Using

$$
\frac{i u(s, i)}{n \cdot \mathbf{u}}=\left(\frac{i u(s, i)}{\sum_{i \in I(s)} i u(s, i)}\right)\left(\frac{\sum_{i \in I(s)} i u(s, i)}{\mathbf{n} \cdot \mathbf{u}}\right)=q(i \mid s) q(s)
$$

we have

$$
\rho(\tau, \theta)=\frac{1}{V_{\mathrm{T}}} \sum_{s^{\prime} \in S} \sum_{s \in S} \sum_{i \in I(s)} v^{\circ}\left(s^{\prime}\right) w\left(s^{\prime} \mid s, i\right) q(i \mid s) q(s)
$$

We now use the regression equation form for $w\left(s^{\prime} \mid s, i\right)$ (eq. (8) of the main text), insert it into eq. (C.4) and obtain

$$
\rho(\tau, \theta)=\frac{1}{V_{\mathrm{T}}} \sum_{s^{\prime} \in S} \sum_{s \in S} \sum_{i \in I(s)} v^{\circ}\left(s^{\prime}\right)\left[w^{\circ}\left(s^{\prime} \mid s\right)-\gamma\left(s^{\prime}, s\right)+\beta\left(s^{\prime}, s\right) \frac{(i-1)}{n(s)-1}+\epsilon_{i}\right] q(i \mid s) q(s),
$$


which becomes

$$
\begin{aligned}
\rho(\tau, \theta)=\frac{1}{V_{\mathrm{T}}}\left[\sum_{s^{\prime} \in S} \sum_{s \in \mathcal{S}} v^{\circ}\left(s^{\prime}\right) w^{\circ}\left(s^{\prime} \mid s\right) q(s) \bigotimes\right. & \\
& \left.\quad+\bigotimes \sum_{s^{\prime} \in S} \sum_{s \in S} \sum_{i \in I(s)} v^{\circ}\left(s^{\prime}\right)\left(-\gamma\left(s^{\prime}, s\right)+\beta\left(s^{\prime}, s\right) \frac{(i-1)}{n(s)-1}\right) q(i \mid s) q(s)\right],
\end{aligned}
$$

since the minimum mean square error used to obtain $\gamma\left(s^{\prime}, s\right)$ and $\beta\left(s^{\prime}, s\right)$ ensures that $\sum_{i \in I(s)} \epsilon_{i} q(i \mid s)=0$ for all $s \in S$ (see Box (1). Using eq. (A.3), the double sum in the first line of eq. (C.5) is seen to be $V_{\mathrm{T}}$, and using the definition of relatedness $r(s)=\sum_{i \in I(s)}[(i-1) /(n(s)-1)] q(i \mid s)$ (see Table 1), we can simplify the sum on the second line of eq. (C.5) using the expression for inclusive fitness (eq. (9) of the main text) to obtain

$$
\rho(\tau, \theta)=\frac{1}{V_{\mathrm{T}}(\tau, \theta)}\left[V_{\mathrm{T}}(\tau, \theta)-1+W_{\mathrm{IF}}(\tau, \theta)\right],
$$

whence

$$
\rho(\tau, \theta)=1+\frac{1}{V_{\mathrm{T}}(\tau, \theta)}\left[W_{\mathrm{IF}}(\tau, \theta)-1\right] .
$$

Since, $V_{\mathrm{T}}(\tau, \theta)>0$, we finally have

$$
\rho(\tau, \theta) \leq 1 \Longleftrightarrow W_{\mathrm{IF}}(\tau, \theta) \leq 1
$$

Hence, a type $\tau$ is uninvadable if it solves $\max _{\tau \in \Theta} W_{\mathrm{IF}}(\tau, \theta)$.

\section{Appendix D: Reproductive numbers}

\section{D.1 Basic reproductive number and expected number of emigrants}

Here, we prove the uninvadability condition expressed in terms of the basic reproductive number (eq. (13) of the main text and Table). According to our notations, the mean matrix of the branching process can be 
decomposed as

$$
\mathrm{A}=\mathrm{P}+\mathrm{D},
$$

where $\mathbf{P}$ is the matrix collecting the $p\left(s^{\prime}, i^{\prime} \mid s, i\right)$ elements and $\mathbf{D}$ is the matrix collecting the $d\left(s^{\prime}, i^{\prime} \mid s, i\right)$ elements (see eq. (11) or Table 11). Then an application of the next generation theorem (Caswell, 2000; Thieme, 2009) shows that

$$
R_{0} \leq 1 \Longleftrightarrow \rho \leq 1
$$

where $R_{0}$ is the leading eigenvalue of the next generation matrix

$$
\mathbf{R}=\mathbf{D}(\mathbf{I}-\mathbf{P})^{-1}
$$

This matrix has leading right eigenvector $\mathbf{u}_{0}$ whose element $u_{0}(s, i)$ is the asymptotic probability that a group initiated by a local lineage starts in state $(s, i)\left(R_{0} \mathbf{u}_{0}=\mathbf{R} \mathbf{u}_{0}\right)$. The elements of $\mathbf{R}$ are

$$
r\left(s^{\prime}, i^{\prime} \mid s, i\right)=\sum_{\zeta \in S} \sum_{j \in I(\zeta)} d\left(s^{\prime}, i^{\prime} \mid \zeta, j\right) t(\zeta, j \mid s, i),
$$

where $t(s, j \mid s, i)$ is the expected number of demographic time steps the mutant lineage spends in state $(\zeta, j)$ over its lifetime in a single group given that the group started in state $(s, i)$. These sojourn times are elements of the "fundamental matrix" $(\mathbf{I}-\mathbf{P})^{-1}$ (Grinstead and Snell, 1997). The interpretation of $r\left(s^{\prime}, i^{\prime} \mid s, i\right)$ is that it gives the total expected number of groups in state $\left(s^{\prime}, i^{\prime}\right)$ produced through dispersal over the lifetime of the mutant lineage in a single group that started in state $(s, i)$.

Using the above, we now rewrite $R_{0}$ using the same line of argument as for lineage fitness. Hence, we first let

$$
\sum_{i^{\prime} \in I\left(s^{\prime}\right)} i^{\prime} d\left(s^{\prime}, i^{\prime} \mid s, i\right)=w_{\mathrm{d}}\left(s^{\prime} \mid s, i\right) i
$$

where $w_{\mathrm{d}}\left(s^{\prime} \mid s, i\right)$ is the total expected successful number of immigrants in groups in state $s^{\prime}$ produced by 
a single mutant in a group in state $(s, i)$. Premultiplying $R_{0} \mathbf{u}_{0}=\mathbf{R u}_{0}$ by $\mathbf{n}$ and using eq. (D.5) entails that

$$
\begin{aligned}
R_{0}(\tau, \theta) & =\frac{1}{\mathbf{n} \cdot \mathbf{u}_{0}} \sum_{s^{\prime} \in s} \sum_{i^{\prime} \in I\left(s^{\prime}\right)} \sum_{s \in s} \sum_{i \in I(s)} i^{\prime} r\left(s^{\prime}, i^{\prime} \mid s, i\right) u_{0}(s, i) \\
& =\frac{1}{\mathbf{n} \cdot \mathbf{u}_{0}} \sum_{s^{\prime} \in S} \sum_{i^{\prime} \in I\left(s^{\prime}\right)} \sum_{s \in S} \sum_{s \in I(s)} \sum_{\zeta \in s} \sum_{j \in I(\zeta)} i^{\prime} d\left(s^{\prime}, i^{\prime} \mid s, j\right) t(s, j \mid s, i) u_{0}(s, i) \\
& =\frac{1}{\mathbf{n} \cdot \mathbf{u}_{0}} \sum_{s^{\prime} \in S} \sum_{s \in S} \sum_{i \in I(s)} \sum_{\zeta \in S} \sum_{j \in I(\zeta)} w_{\mathrm{d}}\left(s^{\prime} \mid \varsigma, j\right) j t(\varsigma, j \mid s, i) u_{0}(s, i) .
\end{aligned}
$$

In order to further simplify $R_{0}$ we set

$$
\bar{t}(\zeta, j)=\sum_{s \in S} \sum_{i \in I(s)} t(\zeta, j \mid s, i) u_{0}(s, i)
$$

which is the average of the expected amount of time the mutant lineage spends in state $(\zeta, j)$ over its lifetime in a single group. We also let

$$
N_{\mathrm{F}}(\tau, \theta)=\mathbf{n} \cdot \mathbf{u}_{0}=\sum_{s \in \mathcal{S}} \sum_{i \in I(s)} i u_{0}(s, i),
$$

which is the expected number of founders of the mutant lineage. By further denoting

$$
R_{\mathrm{m}}(\tau, \theta)=\sum_{s^{\prime} \in \mathcal{S}} \sum_{\zeta \in \mathcal{S}} \sum_{j \in I(\zeta)} w_{\mathrm{d}}\left(s^{\prime} \mid \zeta, j\right) j \bar{t}(\zeta, j),
$$

and inserting into eq. (D.6), we have

$$
R_{0}(\tau, \theta)=\frac{R_{\mathrm{m}}(\tau, \theta)}{N_{\mathrm{F}}(\tau, \theta)}
$$

\section{D.2 Lineage fitness proxy}

We will now rewrite eq. (D.10) in terms of lineage fitness proxy (eq. (15) of the main text). For this, we set

$$
N_{\mathrm{L}}(\tau, \theta)=\sum_{s \in S} \sum_{i \in I(s)} i \bar{t}(s, i)
$$


which is the expected total size of the mutant lineage over its lifetime in a single group. Extending the argument of Mullon and Lehmann (2014, Appendix A), this is also

$$
N_{\mathrm{L}}(\tau, \theta)=N_{\mathrm{F}}(\tau, \theta)+\sum_{s^{\prime} \in S} \sum_{s \in S} \sum_{i \in I(s)} w_{\mathrm{p}}\left(s^{\prime} \mid s, i\right) i \bar{t}(s, i),
$$

since $N_{\mathrm{F}}(\tau, \theta)$ is the expected number of mutant individuals founding a single group and the sum is the expected number of mutant offspring settling locally and produced over the lifetime of the lineage in that group. Subtracting eq. (D.11) from eq. (D.12), inserting into eq. (D.9) and using eq. (6), we can write

$$
\begin{aligned}
R_{\mathrm{m}}(\tau, \theta) & =N_{\mathrm{F}}(\tau, \theta)+\sum_{s^{\prime} \in S} \sum_{s \in \mathcal{S}} \sum_{i \in I(s)} w\left(s^{\prime} \mid s, i\right) i \bar{t}(s, i)-N_{\mathrm{L}}(\tau, \tau) \\
& =N_{\mathrm{F}}(\tau, \theta)+N_{\mathrm{L}}(\tau, \theta) R_{\mathrm{L}}(\tau, \theta)-N_{\mathrm{L}}(\tau, \theta)
\end{aligned}
$$

where the second line follows from using eq. (15). Inserting eq. (15) of the main text and eq. (D.13) into eq. (D.10) gives

$$
R_{0}(\tau, \theta)=1+\frac{N_{\mathrm{L}}(\tau, \theta)}{N_{\mathrm{F}}(\tau, \theta)}\left(R_{\mathrm{L}}(\tau, \theta)-1\right)
$$

which shows that $R_{0}(\tau, \theta) \leq 1 \Longleftrightarrow R_{\mathrm{L}}(\tau, \theta) \leq 1$, whereby

$$
R_{\mathrm{L}}(\tau, \theta) \leq 1 \Longleftrightarrow \rho(\tau, \theta) \leq 1,
$$

\section{Appendix E: Connections to previous work}

We here provide different connections to fitness components that appear in the literature.

\section{E.1 Fitness decomposition: philopatric and dispersed}

We start by further decomposing the two fitness components in eq. (6). First, we can write

$$
w_{\mathrm{p}}\left(s^{\prime} \mid s, i\right)=w_{\mathrm{p}}\left(s^{\prime}, s, i\right) p\left(s^{\prime} \mid s, i\right)
$$


where $p\left(s^{\prime} \mid s, i\right)$ is the probability that a group will be in state $s^{\prime}$ in the offspring generation given that it was in state $(s, i)$ in the parental generation and $w_{\mathrm{p}}\left(s^{\prime}, s, i\right)$ is the expected number of successful philopatric offspring given that the offspring settle in a group in state $s^{\prime}$ and the parent reproduces in a group in state $(s, i)$. We can also write

$$
w_{\mathrm{d}}\left(s^{\prime} \mid s, i\right)=\sum_{x \in S} w_{\mathrm{d}}\left(s^{\prime}, x, s, i\right) k\left(s^{\prime} \mid x, s, i\right) p^{\circ}(x)
$$

where $p^{\circ}(x)$ is the (neutral) probability that a group randomly sampled in the monomorphic resident population is in state $x$. Here, $k\left(s^{\prime} \mid x, s, i\right)$ is the probability that a group that was in state $(x, 0)$ in the parental generation and has been colonized by a mutant descending from a group in state $(s, i)$ will become a group in state $s^{\prime}$ in the offspring generation, and $w_{\mathrm{d}}\left(s^{\prime}, x, s, i\right)$ is the expected number of dispersing offspring that a single mutant produces given that it resides in a group in state $(s, i)$ and given that the group where the offspring settle is in state $s^{\prime}$ in the offspring generation and was in state $x$ in the parental generation (with 0 mutants). The conditional fitness functions $w_{\mathrm{p}}\left(s^{\prime}, s, i\right)$ and $w_{\mathrm{d}}\left(s^{\prime}, x, s, i\right)$ are the elementary individualbased fitness components of models in demographically structured populations (e.g., eqs. 31-32 of Rousset and Ronce, 2004).

We now prove the expressions for the two above conditional expectations (eqs. (E.1)-(E.2)). From Table 1, the first conditional expectation can be written as

$$
w_{\mathrm{p}}\left(s^{\prime} \mid s, i\right)=\frac{1}{i} \sum_{i^{\prime} \in I\left(s^{\prime}\right)} i^{\prime} p\left(s^{\prime}, i^{\prime} \mid s, i\right)=\frac{p\left(s^{\prime} \mid s, i\right)}{i} \sum_{i^{\prime} \in I\left(s^{\prime}\right)} i^{\prime} \frac{p\left(s^{\prime}, i^{\prime} \mid s, i\right)}{\frac{p\left(s^{\prime} \mid s, i\right)}{p\left(i^{\prime} \mid s^{\prime}, s, i\right)}}=p\left(s^{\prime} \mid s, i\right) w_{\mathrm{p}}\left(s^{\prime}, s, i\right),
$$

where $p\left(i^{\prime} \mid s^{\prime}, s, i\right)$ is the probability that a group will have $i^{\prime}$ mutants in the offspring generation given that it is in state $(s, i)$ in the parental generation and in state $s^{\prime}$ in the offspring generation. Here, we used

$$
w_{\mathrm{p}}\left(s^{\prime}, s, i\right) i=\sum_{i^{\prime} \in I\left(s^{\prime}\right)} i^{\prime} p\left(i^{\prime} \mid s^{\prime}, s, i\right)
$$

where $w_{\mathrm{p}}\left(s^{\prime}, s, i\right)$ the expected number of successful philopatric offspring that a single mutant produces given that it resides in a group in state $(s, i)$ and that the group state in the offspring generation is $s^{\prime}$. 
From Table 1, the second conditional expectation is

$$
w_{\mathrm{d}}\left(s^{\prime} \mid s, i\right)=\frac{1}{i} \sum_{i^{\prime} \in I(x)} i^{\prime} d\left(s^{\prime}, i^{\prime} \mid s, i\right),
$$

where, conditioning on the state of the group in the parental generation where the offspring disperse to, we can write

$$
d\left(s^{\prime}, i^{\prime} \mid s, i\right)=\sum_{x \in \mathcal{S}} d\left(s^{\prime}, i^{\prime} \mid x, s, i\right) p^{\circ}(x)=\sum_{x \in \mathcal{S}} \frac{\frac{d\left(s^{\prime}, i^{\prime} \mid x, s, i\right)}{k\left(s^{\prime} \mid x, s, i\right)}}{d\left(i^{\prime} \mid s^{\prime}, x, s, i\right)} k\left(s^{\prime} \mid x, s, i\right) p^{\circ}(x) .
$$

Here, we used in the conditioning the neutral probability $p^{\circ}(x)$ that a group randomly sampled in the monomorphic resident population is in demographic state $x$, since dispersing offspring can only land in a group whose state in the parental generation is determined by the resident dynamics. The term $d\left(s^{\prime}, i^{\prime} \mid\right.$ $x, s, i)$ is the expected number of groups in $\left(s^{\prime}, i^{\prime}\right)$ produced by a group in state $\left(s^{\prime}, i^{\prime}\right)$ and given that they were in state $(0, x)$ in the parental generation (with 0 mutants). We now let

$$
d\left(i^{\prime} \mid s^{\prime}, x, s, i\right)=\frac{d\left(s^{\prime}, i^{\prime} \mid x, s, i\right)}{k\left(s^{\prime} \mid x, s, i\right)}
$$

where $k\left(s^{\prime} \mid x, s, i\right)$ is the probability that a group will be in state $s^{\prime}$ in the offspring generation, given that it was in state $(x, 0)$ in the parental generation and has been colonized by a mutant descending from a group in state $(s, i)$. Further we have

$$
w_{\mathrm{d}}\left(s^{\prime}, x, s, i\right)=\frac{1}{i} \sum_{i^{\prime} \in I\left(s^{\prime}\right)} i^{\prime} d\left(i^{\prime} \mid s^{\prime}, x, s, i\right),
$$

which is the expected number of dispersing offspring that a single mutant produces given that it resides in a group in state $(s, i)$ and given that the group where the offspring settle is in demographic state $s^{\prime}$ in the offspring generation and was in state $(x, 0)$ in the parental generation. Substituting into eq. (E.6), we then obtain eq. (E.2). 


\section{E.2 Connection to the direct fitness method}

We now connect our results to the direct fitness approach (Taylor and Frank, 1996; Rousset, 2004), which, formally, consists of computing the selection gradient on a mutant type when mutant phenotypic deviations are small relative to the resident and is sufficient to evaluate the condition of convergence stability under essentially all conditions (Rousset, 2004; Lehmann and Rousset, 2014). Hence, results from the direct fitness method should match $\partial \rho(\tau, \theta) / \partial \tau$ when the type space is real valued and one dimensional $(\Theta=\mathbb{R})$, which we henceforth assume.

\section{E.3 Sensitivity of the growth rate}

To prove the connection to the direct fitness approach we first derive a generic expression for the growth rate sensitivity $\partial \rho(\tau, \theta) / \partial \tau$ under our model assumptions. To that aim, we rewrite the growth rate by using eq. (C.4) as

$$
\rho(\tau, \theta)=\sum_{s^{\prime} \in \mathcal{S}} \sum_{s \in S} \sum_{i \in I(s)} v^{\circ}\left(s^{\prime}\right) w\left(s^{\prime} \mid s, i\right) q(i \mid s) q_{\mathrm{v}}(s)
$$

where

$$
q_{\mathrm{v}}(s)=\frac{q(s)}{V_{\mathrm{T}}}
$$

Since, $v^{\circ}\left(s^{\prime}\right)$ depends only on the resident, we have

$$
\frac{\partial \rho(\tau, \theta)}{\partial \tau}=\sum_{s^{\prime} \in S} \sum_{s \in S} \sum_{i \in I(s)} v^{\circ}\left(s^{\prime}\right)\left[\frac{\partial w\left(s^{\prime} \mid s\right)}{\partial \tau} q^{\circ}(i \mid s) q_{\mathrm{v}}^{\circ}(s)+w^{\circ}\left(s^{\prime} \mid s, i\right) \frac{\partial\left[q(i \mid s) q_{\mathrm{v}}(s)\right]}{\partial \tau}\right],
$$

where all derivatives, here and throughout, are evaluated at $\tau=\theta$. Using the neutral reproductive values (eq. (A.3)), we have

$$
\sum_{s^{\prime} \in S} \sum_{s \in S} \sum_{i \in I(s)} v^{\circ}\left(s^{\prime}\right) w^{\circ}\left(s^{\prime} \mid s\right) \frac{\partial\left[q(i \mid s) q_{\mathrm{v}}(s)\right]}{\partial \tau}=\sum_{s \in S} \sum_{i \in I(s)} v^{\circ}(s) \frac{\partial\left[q(i \mid s) q_{\mathrm{v}}(s)\right]}{\partial \tau}
$$


Further, we have

$$
\begin{aligned}
\sum_{s \in S} \sum_{i \in I(s)} v^{\circ}(s) \frac{\partial\left[q(i \mid s) q_{\mathrm{v}}(s)\right]}{\partial \tau} & =\frac{\partial}{\partial \tau}\left[\sum_{s \in S} \sum_{i \in I(s)} v^{\circ}(s) q(i \mid s) q_{\mathrm{v}}(s)\right] \\
& =\frac{\partial}{\partial \tau}\left[\sum_{s \in S} \sum_{i \in I(s)} v^{\circ}(s) \frac{i u(i, s)}{\mathbf{v}^{\circ} \cdot \mathbf{u}}\right] \\
& =\frac{\partial}{\partial \tau}(1) \\
& =0 .
\end{aligned}
$$

Hence, substituting eq. (E.13) into eq. (E.11) using eq. (E.10) gives

$$
\frac{\partial \rho(\tau, \theta)}{\partial \tau}=\frac{1}{V_{\mathrm{T}}^{\circ}} \sum_{s^{\prime} \in S} \sum_{s \in S} v^{\circ}\left(s^{\prime}\right)\left[\sum_{i \in I(s)} \frac{\partial w\left(s^{\prime} \mid s, i\right)}{\partial \tau} q^{\circ}(i \mid s)\right] q^{\circ}(s),
$$

where without loss of generality we can normalize the elements $v^{\circ}\left(s^{\prime}\right)$ such that $V_{\mathrm{T}}^{\circ}=1$.

Note that $w\left(s^{\prime} \mid s, i\right)$ is the personal fitness of a mutant with phenotype $\tau$ when its group members consist of $i-1$ individuals with phenotype $\tau$ and $n(s)-i$ individuals with phenotype $\theta$. Thus, we can write

$$
\frac{\partial w\left(s^{\prime} \mid s, i\right)}{\partial \tau}=\frac{\partial w\left(s^{\prime} \mid s, \tau_{j}, \tau_{-j}\right)}{\partial \tau_{j}}+(n(s)-1) \frac{\partial w\left(s^{\prime} \mid s, \tau_{j}, \tau_{-j}\right)}{\partial \tau_{k}} \frac{i-1}{n(s)-1}
$$

where $\tau_{-j}=\left(\tau_{1}, \ldots, \tau_{j-1}, \tau_{j+1}, \ldots \tau_{n(s)-1}\right)$ is the vector collecting the phenotypes of the neighbors of an individual $j$ and $k \neq j$. Substituting into eq. (E.14), setting $V_{\mathrm{T}}^{\circ}=1$, and using the definition of relatedness given in the Table 1 gives

$$
\frac{\partial \rho(\tau, \theta)}{\partial \tau}=\sum_{s^{\prime} \in S} \sum_{s \in S} v^{\circ}\left(s^{\prime}\right)\left[\frac{\partial w\left(s^{\prime} \mid s, \tau_{j}, \tau_{-j}\right)}{\partial \tau_{j}}+(n(s)-1) \frac{\partial w\left(s^{\prime} \mid s, \tau_{j}, \tau_{-j}\right)}{\partial \tau_{k}} r^{\circ}(s)\right] q^{\circ}(s) .
$$

\section{E.3.1 Connection to direct fitness method results}

Here, we prove that eq. (E.16) returns exactly eqs. 26-27 of Rousset and Ronce (2004) when states are population sizes and each individuals migrates independently from each other. This proves that we recover in general the results obtained by the direct fitness method since the results of Rousset and Ronce (2004) generalize those of Taylor and Frank (1996) to demographically structured populations. 
In order to show the connection, we need to prove that

$$
q^{\circ}(s)=\frac{p^{\circ}(s) n(s)}{\bar{n}^{\circ}}
$$

where $\bar{n}^{\circ}=\sum_{s \in S s} n(s) p^{\circ}(s)$ is the average group size in a monomorphic $\theta$ population. For this, we first note that from the definition of $q(s)$ (Table 1), we have

$$
\begin{aligned}
q^{\circ}\left(s^{\prime}\right) & =\sum_{i^{\prime} \in I\left(s^{\prime}\right)} q^{\circ}\left(s^{\prime}, i^{\prime}\right) \\
& =\sum_{i^{\prime} \in I\left(s^{\prime}\right)} \frac{i^{\prime} u^{\circ}\left(s^{\prime}, i^{\prime}\right)}{\mathbf{n} \cdot \mathbf{u}^{\circ}} \\
& =\frac{1}{\mathbf{n} \cdot \mathbf{u}^{\circ}} \sum_{i^{\prime} \in I\left(s^{\prime}\right)} \sum_{s \in S} \sum_{i \in I(s)} i^{\prime} a\left(s^{\prime}, i^{\prime} \mid s, i\right) u^{\circ}(s, i) \\
& =\frac{1}{\mathbf{n} \cdot \mathbf{u}^{\circ}} \sum_{s \in S} \sum_{i \in I(s)} w\left(s^{\prime}, s\right) i u^{\circ}(s, i),
\end{aligned}
$$

which yields

$$
q^{\circ}\left(s^{\prime}\right)=\sum_{s \in S} w^{\circ}\left(s^{\prime} \mid s\right) q^{\circ}(s)
$$

and shows that the vector collecting the $q^{\circ}(s)$ is a right unit eigenvector of the matrix with elements $w^{\circ}\left(s^{\prime} \mid\right.$ $s)$. Let us now substitute the trial solution $q^{\circ}(s)=n(s) p^{\circ}(s) / \bar{n}^{\circ}$ into eq. (E.19), whereby

$$
n\left(s^{\prime}\right) p^{\circ}\left(s^{\prime}\right)=\sum_{s \in S} w^{\circ}\left(s^{\prime} \mid s\right) n(s) p^{\circ}(s)
$$

The right hand side is the total expected number of successful offspring in groups in state $s^{\prime}$ that descend from a randomly sampled group in the population. At stationarity this must be equal to $n\left(s^{\prime}\right) p^{\circ}\left(s^{\prime}\right)$, since $p^{\circ}\left(s^{\prime}\right)$ is the probability of sampling a group in state $s^{\prime}$ and $n\left(s^{\prime}\right)$ is the number of successful offspring in that group. Hence, $q^{\circ}(s)=n(s) p^{\circ}(s) / \bar{n}^{\circ}$ satisfies eq. (E.19) and eq. (E.17) holds.

We now expand eq. (E.16) by using the decomposition of personal fitness $w\left(s^{\prime} \mid s, i\right)=w_{\mathrm{p}}\left(s^{\prime} \mid s, i\right)+$ $w_{\mathrm{d}}\left(s^{\prime} \mid s, i\right)$ (eq. (6) of the main text), which allows us to write

$$
\frac{\partial w\left(s^{\prime} \mid s, \tau_{j}, \tau_{-j}\right)}{\partial \tau_{j}}=\frac{\partial w_{\mathrm{p}}\left(s^{\prime} \mid s, \tau_{j}, \tau_{-j}\right)}{\partial \tau_{j}}+\frac{\partial w_{\mathrm{d}}\left(s^{\prime} \mid s, \tau_{j}, \tau_{-j}\right)}{\partial \tau_{j}}
$$


Each of these component will be further expanded by using eqs. (E.1)-(E.2). For the philopatric component, from eq. (E.1) we can write

$$
\frac{\partial w_{\mathrm{p}}\left(s^{\prime} \mid s, \tau_{j}, \tau_{-j}\right)}{\partial \tau_{j}}=\frac{\partial w_{\mathrm{p}}\left(s^{\prime}, s, \tau_{j}, \tau_{-j}\right)}{\partial \tau_{j}} p^{\circ}\left(s^{\prime} \mid s\right)+w_{\mathrm{p}}^{\circ}\left(s^{\prime}, s\right) \frac{\partial p\left(s^{\prime} \mid s, \tau\right)}{\partial \tau_{j}},
$$

where $\tau=\left(\tau_{1}, \ldots, \tau_{n(s)-1}\right)$. For a neighbour $k \neq j$ of a focal mutant $j$ we have

$$
\frac{\partial w_{\mathrm{p}}\left(s^{\prime} \mid s, \tau_{j}, \tau_{-j}\right)}{\partial \tau_{k}}=\frac{\partial w_{\mathrm{p}}\left(s^{\prime}, s, \tau_{j}, \tau_{-j}\right)}{\partial \tau_{k}} p^{\circ}\left(s^{\prime} \mid s\right)+w_{\mathrm{p}}^{\circ}\left(s^{\prime}, s\right) \frac{\partial p\left(s^{\prime} \mid s, \tau\right)}{\partial \tau_{k}} .
$$

In order to expand the dispersal component in eq. (E.21), we follow the assumption of Rousset and Ronce, 2004 that the composition of a natal group of mutants does not affect the transition probability of other groups (owing to the fact that individuals migrate independently from each other) and set $k\left(s^{\prime} \mid\right.$ $\varsigma, s, i)=p^{\circ}\left(s^{\prime} \mid \varsigma\right)$ in eq. (E.2). Then, we can write

$$
\frac{\partial w_{\mathrm{d}}\left(s^{\prime} \mid s, \tau_{j}, \tau_{-j}\right)}{\partial \tau_{j}}=\sum_{\zeta \in \mathcal{S}} \frac{\partial w_{\mathrm{d}}\left(s^{\prime}, \zeta, s, \tau_{j}, \tau_{-j}\right)}{\partial \tau_{j}} p^{\circ}\left(s^{\prime} \mid \zeta\right) p^{\circ}(\zeta)
$$

and for $k \neq j$

$$
\frac{\partial w_{\mathrm{d}}\left(s^{\prime} \mid s, \tau_{j}, \tau_{-j}\right)}{\partial \tau_{k}}=\sum_{\varsigma \in \mathcal{S}} \frac{\partial w_{\mathrm{d}}\left(s^{\prime}, \varsigma, s, \tau_{j}, \tau_{-j}\right)}{\partial \tau_{k}} p^{\circ}\left(s^{\prime} \mid \varsigma\right) p^{\circ}(\varsigma)
$$

Substituting eqs. (E.21) $-(\overline{E .25})$ into eq. (E.16) yields

$$
\frac{\partial \rho(\tau, \theta)}{\partial \tau}=\sum_{s \in S}\left[S_{\mathrm{f}}(s)+S_{\mathrm{Pr}}(s)\right] \frac{n(s) p^{\circ}(s)}{\bar{n}^{\circ}}
$$

where

$$
\begin{aligned}
S_{\mathrm{f}}(s) & =\sum_{s^{\prime} \in \mathcal{S}} v^{\circ}\left(s^{\prime}\right)\left[\left(\frac{\partial w_{\mathrm{p}}\left(s^{\prime}, s, \tau_{j}, \tau_{-j}\right)}{\partial \tau_{j}}+\frac{\partial w_{\mathrm{p}}\left(s^{\prime}, s, \tau_{j}, \tau_{-j}\right)}{\partial \tau_{j}}(n(s)-1) r^{\circ}(s)\right) p^{\circ}\left(s^{\prime} \mid s\right) \bigotimes\right. \\
& \left.+\bigotimes \sum_{\zeta \in \mathcal{S}}\left(\frac{\partial w_{\mathrm{d}}\left(s^{\prime}, \zeta, s, \tau_{j}, \tau_{-j}\right)}{\partial \tau_{j}}+\frac{\partial w_{\mathrm{d}}\left(s^{\prime}, \varsigma_{,}, s, \tau_{j}, \tau_{-j}\right)}{\partial \tau_{j}}(n(s)-1) r^{\circ}(s)\right) p^{\circ}\left(s^{\prime} \mid \varsigma\right) p^{\circ}(\varsigma)\right],
\end{aligned}
$$


and

$$
S_{\operatorname{Pr}}(s)=\sum_{s^{\prime} \in S} v^{\circ}\left(s^{\prime}\right)\left[w_{\mathrm{p}}^{\circ}\left(s^{\prime}, s\right) \frac{\partial p\left(s^{\prime} \mid s, \tau\right)}{\partial \tau_{j}}\left[1+(n(s)-1) r^{\circ}(s)\right]\right]
$$

If we let $s$ be group size and set $n(s)=s$, then eqs. (E.27) $-(\overline{E .28})$ are proportional to eqs. (A.33)-(A.36) of Lehmann and Rousset (2010). If we multiply eq. (E.26) by $n\left(s^{\prime}\right) / n\left(s^{\prime}\right)$ and use class reproductive values $\alpha^{\circ}\left(s^{\prime}\right)=v^{\circ}\left(s^{\prime}\right) n\left(s^{\prime}\right)$ and the definition of frequency functions of Rousset and Ronce (2004, eqs. 33-34), then eqs. (E.27)-(E.28) are proportional to eqs. (26)-(27) of Rousset and Ronce (2004).

\section{Appendix F: Fixed number of age or stage classes}

We here consider a situation where there is a uniform demography, where each group is of constant size but now each individual belongs to one of a set of fixed classes where the set of class is given by $C=\left\{1, \ldots, n_{\mathrm{c}}\right\}$. An example would age structure due to overlapping generations or different castes of social insects like workers and queens.

Let $\mathbf{i}=\left(i_{1}, \ldots, i_{n_{\mathrm{c}}}\right) \in i$ be the vector of the number of mutant alleles of type $\tau$ in class 1 to $n_{\mathrm{c}}$ in a group where $i$ is the set of possible configurations. Let $I=\left(I_{1} \times \cdots \times I_{n_{c}}\right) \quad 0$ where $I_{x}=\left\{0,1, \ldots, n_{x}\right\}$ is set of the number of mutant alleles in class $x$ and $n_{x}$ is the number of individuals in that class. We remove the all zero state $\mathbf{0}$ from $I$ so that we only track states with at least one mutant in some class. Let $\mathrm{A}$ be the matrix with elements $a\left(\mathbf{i}^{\prime} \mid \mathbf{i}\right)$ giving the expected number of groups in state $i^{\prime}$ produced by a focal group in state $\mathbf{i}^{\prime}$. Further, let $\mathbf{n}$ be the vector collecting the total number of mutant individuals for each state; i.e., the $\mathbf{i}$-th state of $\mathbf{n}$ is given by $x(\mathbf{i})=\sum_{y \in C} i_{y}$.

We now prove the expression for lineage fitness (e.g., eq. 5) for this model and proceed in the same way as in Appendices $A-\mathrm{G}$. Hence, we first note that

$$
\sum_{\mathbf{i}^{\prime} \in i} i_{y}^{\prime} a\left(\mathbf{i}^{\prime} \mid \mathbf{i}\right)=\sum_{x \in C} w(y, x, \mathbf{i}) i_{x}
$$

where $w(y, x, \mathbf{i})$ is the expected number of class $y$ offspring produced by a class $x$ mutant when in a group 
in state i. Now, from $\mathbf{n} \cdot \rho \mathbf{u}=(\mathbf{n} \cdot$ Au $)$ and eq. (F.1), we have

$$
\begin{aligned}
\rho(\tau, \theta) & =\frac{1}{\mathbf{n} \cdot \mathbf{u}} \sum_{\mathbf{i}^{\prime} \in I} \sum_{\mathbf{i} \in I} x\left(\mathbf{i}^{\prime}\right) a\left(\mathbf{i}^{\prime} \mid \mathbf{i}\right) u(\mathbf{i}) \\
& =\frac{1}{\mathbf{n} \cdot \mathbf{u}} \sum_{\mathbf{i}^{\prime} \in I} \sum_{\mathbf{i} \in I} \sum_{y \in C} i_{y}^{\prime} a\left(\mathbf{i}^{\prime} \mid \mathbf{i}\right) u(\mathbf{i}) \\
& =\frac{1}{\mathbf{n} \cdot \mathbf{u}} \sum_{\mathbf{i} \in I} \sum_{y \in C} \sum_{x \in C} w(y, x, \mathbf{i}) i_{x} u(\mathbf{i}) \\
& =\sum_{\mathbf{i} \in I} \sum_{y \in C} \sum_{x \in C} w(y, x, \mathbf{i}) q(x, \mathbf{i})
\end{aligned}
$$

where

$$
q(x, \mathbf{i})=\frac{i_{x} u(\mathbf{i})}{\mathbf{n} \cdot \mathbf{u}}
$$

which satisfies $\sum_{x \in C} \sum_{\mathbf{i} \in I} q_{x}(\mathbf{i})=1$. Defining lineage fitness as

$$
W(\tau, \theta)=\sum_{\mathbf{i} \in I} \sum_{y \in C} \sum_{x \in C} w(y, x, \mathbf{i}) q(x, \mathbf{i}),
$$

eq. (F.2) shows that $\rho(\tau, \theta)=W(\tau, \theta)$, which is the same result as eq. (ఫ).

Second, we derive an expression for the inclusive fitness $W_{\mathrm{IF}}(\tau, \theta)$. Inclusive fitness requires that we calculate reproductive values, so we gather into the vector $\mathbf{v}^{\circ}$ the elements

$$
v^{\circ}(\mathbf{i})=\sum_{x \in C} i_{x} v^{\circ}(x),
$$

where $v^{\circ}(x)$ is the reproductive value of an individual in class $x$. Now, from $\mathbf{v}^{\circ} \cdot \rho \mathbf{u}=\left(\mathbf{v}^{\circ} \cdot \mathbf{A u}\right)$ and eq. (F.5), 
we have

$$
\begin{aligned}
\rho(\tau, \theta) & =\frac{1}{\mathbf{v}^{\circ} \cdot \mathbf{u}} \sum_{\mathbf{i}^{\prime} \in I} \sum_{\mathbf{i} \in I} v^{\circ}\left(\mathbf{i}^{\prime}\right) a\left(\mathbf{i}^{\prime} \mid \mathbf{i}\right) u(\mathbf{i}) \\
& =\frac{1}{\mathbf{v}^{\circ} \cdot \mathbf{u}} \sum_{y \in C} \sum_{\mathbf{i}^{\prime} \in I} \sum_{\mathbf{i} \in I} i_{y}^{\prime} v^{\circ}(y) a\left(\mathbf{i}^{\prime} \mid \mathbf{i}\right) u(\mathbf{i}) \\
& =\frac{1}{\mathbf{v}^{\circ} \cdot \mathbf{u}} \sum_{y \in C} \sum_{x \in C} \sum_{\mathbf{i} \in I} v^{\circ}(y) w(y, x, \mathbf{i}) i_{x} u(\mathbf{i}) \\
& =\frac{1}{V_{\mathrm{T}}} \sum_{y \in C} \sum_{x \in C} \sum_{\mathbf{i} \in I} v^{\circ}(y) w(y, x, \mathbf{i}) q(x, \mathbf{i}),
\end{aligned}
$$

where

$$
V_{\mathrm{T}}=\frac{\mathbf{v}^{\circ} \cdot \mathbf{u}}{\mathbf{n} \cdot \mathbf{u}}=\sum_{x \in C} v^{\circ}(x) q(x)
$$

and $q(x)=\sum_{\mathbf{i} \in I} q(x, \mathbf{i})$ is the probability of sampling a lineage member in class $x$. Suppose we now form a weighted multiple regression

$$
w(y, x, \mathbf{i})=w^{\circ}(y, x)-\gamma(y, x)+\beta_{x}(y, x) \frac{i_{x}-1}{n_{x}-1}+\sum_{z \in C} \beta_{z}(y, x) \frac{i_{z}}{n_{z}}+e_{x}(\mathbf{i})
$$

and least square fit $\gamma$ and the $\beta$ 's by minimizing

$$
\sum_{\mathbf{i} \in I} e_{x}(\mathbf{i})^{2} q(x, \mathbf{i}) / q(x)
$$

where the weights are given by $q_{x}(\mathbf{i}) / q_{x}$. This procedure guarantees that the weighted sum of errors is zero, or that $\sum_{\mathbf{i} \in I}=e_{x}(\mathbf{i})\left(q_{x}(\mathbf{i}) / q_{x}\right)=0$. Let us further define

$$
q\left(i_{y} \mid x\right)=\sum_{\mathbf{i} \in I\left(i_{y}\right)} q(x, \mathbf{i}) / q(x)
$$

where $I\left(i_{y}\right)$ denotes the elements of the set $I$ whose number of class $y$ mutants is equal to $i_{y}$. Then, we can interpret $q\left(i_{y} \mid x\right)$ as the probability that there are $i_{y}$ mutants in class $y$ given that a mutant has a sampled a 
mutant in class $x$. Substituting all this into eq. (F.6), we have

$$
\begin{aligned}
\rho(\tau, \theta)=\frac{1}{V_{\mathrm{T}}(\tau, \theta)} \sum_{y \in C} & \sum_{x \in C} v^{\circ}(y)\left[\bigotimes w^{\circ}(y, x)-\gamma(y, x)\right. \\
& \left.+\sum_{i_{x} \in I_{x}^{+}} \beta_{x}(y, x) \frac{i_{x}-1}{n_{x}-1} q\left(i_{x} \mid x\right)+\sum_{z \in C} \sum_{i_{z} \in I_{z}^{+}} \beta_{z}(y, x) \frac{i_{z}}{n_{z}} q\left(i_{z} \mid x\right)\right] \bowtie q(x)
\end{aligned}
$$

where we only sum over the elements $I_{x}^{+}=\left\{1, \ldots, n_{x}\right\}$ in the first sum of the second line since $w(y, x, \mathbf{i})=0$ for all $i_{x}=0$ (the second sum in the second line uses $i_{z}^{+}$for ease of notation).

Let us now define inclusive fitness as

$$
W_{\mathrm{IF}}(\tau, \theta)=1+\sum_{y \in C} \sum_{x \in C} v^{\circ}(y)\left[-\gamma(y, x)+\sum_{z \in C} \beta_{z}(y, x) r(z \mid x)\right] q(x),
$$

where

$$
r(z \mid x)=\sum_{i_{z} \in I_{z}^{+}}\left[\delta_{z x} \frac{i_{x}-1}{n_{x}-1}+\left(1-\delta_{z x}\right) \frac{i_{z}}{n_{z}}\right] q\left(i_{z} \mid x\right)
$$

is the probability that, conditional on being sampled in class $x$, an individual carrying the mutant experiences a randomly sampled neighbour in class $z$ that also carries the mutant allele, and where $\delta_{z x}$ is the Kronecker Delta $\left(\delta_{z x}=1\right.$ if $z=x$, zero otherwise). Substituting eqs. (F.12) $-($ F.13) into eq. (F.11) and using the definition of reproductive value we obtain

$$
\rho(\tau, \theta)=\frac{1}{V_{\mathrm{T}}(\tau, \theta)}\left[V_{\mathrm{T}}(\tau, \theta)+W_{\mathrm{IF}}(\tau, \theta)\right]
$$

whereby

$$
\rho(\tau, \theta) \leq 1 \Longleftrightarrow W_{\mathrm{IF}}(\tau, \theta) \leq 1
$$

